Review article

\title{
Blood-brain barrier regulation in psychiatric disorders
}

\author{
John Kealy ${ }^{\mathrm{a}, \mathrm{b}, 1}$, Chris Greene ${ }^{\mathrm{a}, *, 1}$, Matthew Campbell ${ }^{\mathrm{a}, *}$ \\ a Smurfit Institute of Genetics, Trinity College Dublin, Dublin 2, Ireland \\ ${ }^{\mathrm{b}}$ School of Biochemistry \& Immunology, Trinity Biomedical Sciences Institute, Trinity College Dublin, Dublin 2, Ireland
}

A R T I C L E I N F O

\section{Keywords:}

Endothelial cell

Tight junction

Claudin-5

Schizophrenia

Autism spectrum disorder

Depression

\begin{abstract}
A B S T R A C T
The blood-brain barrier (BBB) is a dynamic interface between the peripheral blood supply and the cerebral parenchyma, controlling the transport of material to and from the brain. Tight junctions between the endothelial cells of the cerebral microvasculature limit the passage of large, negatively charged molecules via paracellular diffusion whereas transcellular transportation across the endothelial cell is controlled by a number of mechanisms including transporter proteins, endocytosis, and diffusion. Here, we review the evidence that perturbation of these processes may underlie the development of psychiatric disorders including schizophrenia, autism spectrum disorder (ASD), and affective disorders. Increased permeability of the BBB appears to be a common factor in these disorders, leading to increased infiltration of peripheral material into the brain culminating in neuroinflammation and oxidative stress. However, although there is no common mechanism underpinning BBB dysfunction even within each particular disorder, the tight junction protein claudin-5 may be a clinically relevant target given that both clinical and pre-clinical research has linked it to schizophrenia, ASD, and depression. Additionally, we discuss the clinical significance of the BBB in diagnosis (genetic markers, dynamic contrast-enhanced-magnetic resonance imaging, and blood biomarkers) and in treatment (drug delivery).
\end{abstract}

\section{Introduction}

The central nervous system (CNS) consists of the brain and spinal cord, which together regulate the body's response to internal and external stimuli. Central to this function is the neuron, a terminally differentiated electrically excitable cell, which requires a fine control of both electrophysiological and chemical signals to function efficiently. Given the lack of regenerative capacities of neurons, maintaining a constant state of homeostasis in the CNS is essential for the health and integrity of neurons. To maintain optimal synaptic signalling, a tight control of the microenvironment is required to efficiently process the vast array of information received by the CNS. Indeed, while the brain accounts for just $2 \%$ of bodily mass, it expends up to $20 \%$ of the body's energy [1]. However, as the CNS has no local energy reserves, its energy needs are met by the cerebral microvasculature. The combined surface area of microvessels in the brain is $150-200 \mathrm{~cm}^{2} / \mathrm{g}$ of tissue, equating to $\sim 15-20 \mathrm{~m}^{2}$ per adult human brain resulting in a dense mesh-like network of vessels providing blood flow to all brain regions [2]. Small, branched vessels permeate the cerebral parenchyma and these microcapillaries are about $3 \mu \mathrm{m}$ in diameter and are usually found within $25 \mu \mathrm{m}$ of a neuron to ensure that neurons are intimately linked with their blood supply [3,4]. This ensures an efficient supply of oxygenated blood and a constant flow of energy substrates, in addition to a mode of clearing unwanted material away from the brain [5]. However, cerebral blood vessels differ from the rest of the body with the presence of the blood-brain barrier (BBB), the interface between the cerebral parenchyma and the peripheral vasculature. The BBB is typically seen as the brain's first line of defence against potentially harmful material

\footnotetext{
Abbreviations: 22q11DS, 22q11 deletion syndrome; AQP4, aquaporin-4; ASD, autism spectrum disorders; BBB, blood-brain barrier; CBF, cerebral blood flow; CK-BB,

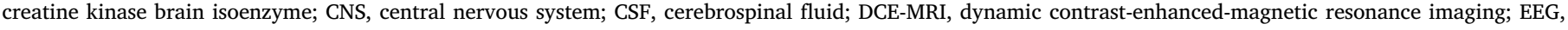

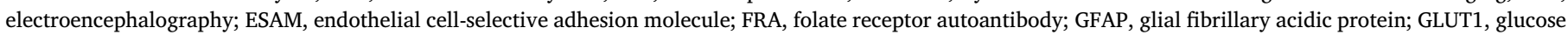

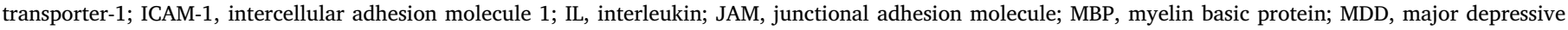

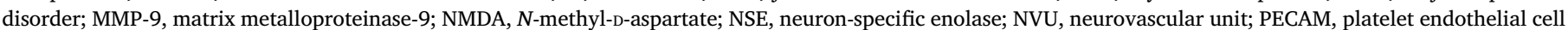

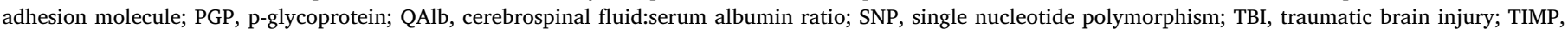

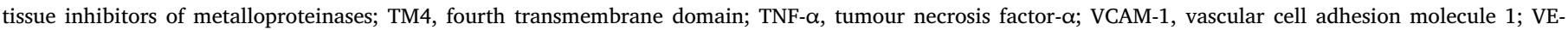
Cadherin, vascular endothelial cadherin; VEGF, vascular endothelial growth factor; ZO, zonula occludens

* Corresponding authors.

E-mail addresses: greenech@tcd.ie (C. Greene), matthew.campbell@tcd.ie (M. Campbell).

${ }^{1}$ These authors contributed equally to this manuscript.
} 
circulating in the blood [6]. From a neuropsychiatric perspective, this unfortunately includes most neuroactive compounds which cannot gain access to the brain and therefore are therapeutically ineffective. This has implications for drug development as researchers attempt to synthesise clinically effective compounds that can cross the BBB or to use various methods to help drugs pass through the BBB [7].

In recent years, the role of the $\mathrm{BBB}$ in neurological disorders has been expanded greatly. The BBB has been shown to be a dynamic boundary between the brain and the periphery, rather than the traditional view of a static "sieve", and the BBB is known to change over the average human lifespan [8]. Pathological changes in BBB structure and permeability have been identified in a range of disorders including Alzheimer's disease [9-11], epilepsy, traumatic brain injury (TBI) [12], chronic traumatic encephalopathy (CTE) [13], and a number of psychiatric disorders (see Section 3). This review will summarise the basic biology underpinning BBB function, provide evidence for BBB dysfunction in psychiatric disorders, and how BBB biology can be incorporated clinically into treatments and diagnostic tools for these disorders.

\section{The structure and function of the BBB}

The BBB, positioned along blood vessels of the CNS, is one of a few selective and tightly regulated barriers and reflects the brain's critical roles in cognitive function, maintaining homeostasis and strictly coordinating the functions of peripheral organs. The BBB constitutes part of the neurovascular unit (NVU), a conglomeration of different cell types including neurons, astrocytes, microglia, pericytes, and endothelial cells of the cerebral microvasculature (Fig. 1A ). The various cell types of the NVU work in concert to ensure that there is even and efficient blood supply to the brain (known as autoregulation), and that increased metabolic demand due to neuronal activation is rapidly matched by an increase in cerebral blood flow (CBF; known as hyperaemia) [14]. In performing these functions, the BBB is vital in regulating the exchange of ions and nutrients between the blood and brain but also to protect delicate neural tissue from potentially damaging blood-borne agents such as pathogens, immune cells and anaphylatoxins [15]. Additionally, the brain endothelium itself secretes $\sim 200 \mathrm{ml}$ of fresh interstitial fluid per day to create an ideal ionic environment for neural function [16].

To maintain homeostasis within the CNS, the BBB has evolved at the level of the cerebral microvasculature (Fig. 1B). Lining blood vessels of the CNS are endothelial cells which separate the peripheral blood from brain tissue. Owing to their specialised function, CNS endothelial cells are distinct from endothelial cells of the periphery in several ways and possess numerous specialisations including:

i) BBB-specific proteins to control the entry and exit of metabolites across cells (transcellular pathway) [17].

ii) Enrichment of highly electrical resistant tight junction proteins to limit the flow of material between adjacent endothelial cells (paracellular pathway).

iii) Absence of fenestrations which are pores to allow the rapid exchange of molecules between blood and tissue in peripheral endothelial cells [18].

iv) Low rate of vesicular transport (transcytosis) to prevent transport of large hydrophilic molecules to the CNS $[15,19]$.

v) BBB peptide transport systems including receptor-mediated $[20,21]$, adsorptive-mediated [22] and carrier-mediated transcytosis $[15,17]$ for the transport of large molecular weight solutes such as proteins and peptides.

vi) Increased mitochondrial numbers to facilitate higher energy expenditure [23].

The inter-endothelial space of the cerebral microvasculature is characterised by the presence of junctional complexes including the adherens junctions and tight junctions. They form the BBB along with the basement membrane, pericytes, and astrocytic endfeet (forming the glia limitans) to restrict permeability across the BBB [6]. Adherens junctions consist of cadherin and catenin proteins that span the intercellular space between endothelial cells and associate with catenin proteins which in turn bind to cytoskeletal proteins, thus linking cells and regulating cell to cell contacts [24]. The precise role of adherens junctions has yet to be resolved, however it is thought that they are involved in maintaining cellular polarity, providing stability, promoting endothelial cell survival and responding to stimuli via interactions with cadherin or catenin proteins and the actin cytoskeleton [25].

Unlike adherens junctions which are present in all vascular beds, tight junctions are enriched in the endothelium of the brain microvasculature. Tight junctions span the intercellular space and interact with tight junction proteins on adjacent endothelial cells at so called "kissing points" to seal the paracellular space [26]. The binding of tight junction proteins impedes the flow of solutes and ions from the blood to brain and vice-versa, in turn creating a dynamic and highly regulatable barrier system. Tight junctions are primarily composed of claudins and occludin which are linked to the actin cytoskeleton by zonula occludens (ZO) proteins [6]. The presence of the tight junction limits paracellular permeability and maintains polarity by enabling asymmetric distribution of membrane constituents [27].

The major tight junction proteins are the claudins and occludin. Claudins and occludin are integral membrane proteins that consist of four transmembrane domains and two extracellular loops. Despite their similar structure, they share minimal sequence homology. The claudin family of proteins consists of up to 24 members. Interactions between the claudins and occludin and the actin cytoskeleton is mediated by the cytoplasmic ZO proteins on the intracellular domain of the plasma membrane to "tether" the tight junctions to the actin cytoskeleton [28-30]. In addition to the claudins and occludin, enriched at two cell contacts, tricellulin and lipolysis-stimulated lipoprotein receptor have been identified at three cell contacts. Other proteins present within the tight junction system are the junctional adhesion molecules (JAMs). JAMs are integral membrane proteins belonging to the immunoglobulin superfamily, of which several isoforms have been discovered. JAMs form homotypic and heterotypic interactions with JAM family members on opposing endothelial cells and are thought to be involved in leukocyte migration across endothelial cell layers [31,32].

In addition to endothelial cells; pericytes, astrocytes and the acellular basement membrane are also key to maintaining BBB function and integrity. Pericytes are mural cells embedded in the basement membrane and envelop blood vessels of the CNS during embryogenesis [33]. They are vital to BBB formation where they are recruited to endothelial cells through paracrine platelet-derived growth factor $\beta$ (PDGF $\beta$ )PDGF $\beta$ receptor (PDGFR $\beta$ ) communication [33-35]. Indeed, PDGF $\beta$ and PDGFR $\beta$ knockout mice are embryonic lethal and have tight junction dysfunction and increased vascular permeability owing to loss of pericyte coverage $[33,36]$. Pericytes have several functions and regulate $\mathrm{BBB}$ permeability, angiogenesis, $\mathrm{CBF}$ and clearance. Pericytes regulate $\mathrm{BBB}$ permeability by controlling the expression of BBB tight junction and adherens junction proteins $[33,37,38]$. Pericytes can also perform macrophage-like activities. They express scavenger receptors and pericytes in culture can ingest macromolecules including polystyrene beads $[39,40]$. Pericyte dysfunction leads to the extravasation of blood-borne neurotoxic macromolecules and likely plays a role in neurological disorders such as Alzheimer's disease [9,38,41,42] and diabetic retinopathy $[43,44]$. To date, there has been little attention devoted to the role of pericytes in neuropsychiatric disorders.

Barrier properties at the BBB are conferred by highly electrical-resistant tight junction proteins in concert with polarised transporter proteins and surrounding NVU cells that limits the flux of all but the smallest molecules and ions. Without considering any relationships between psychiatric disorders and the $\mathrm{BBB}$, these barrier properties are of clinical significance as from a neuropharmacological point of view, 

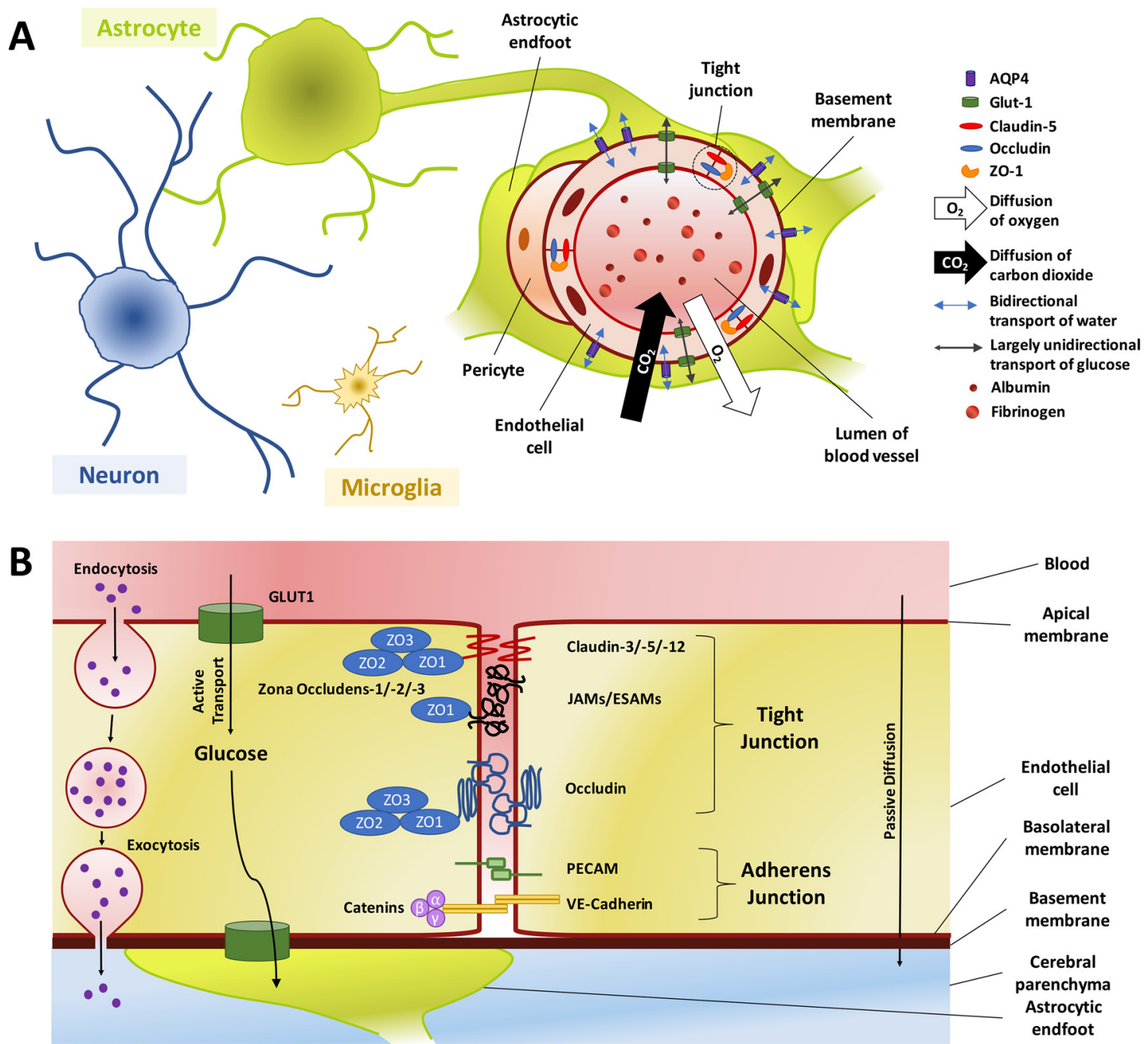

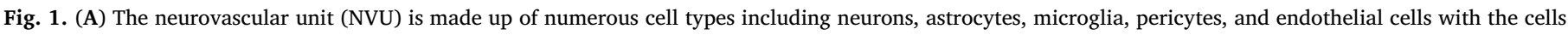

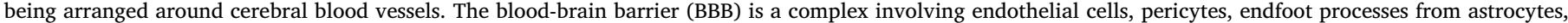

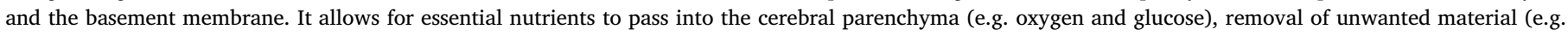

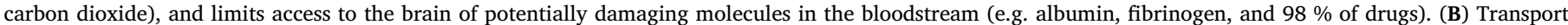

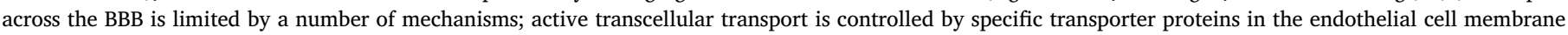

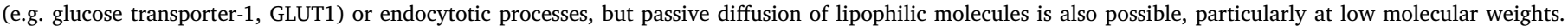

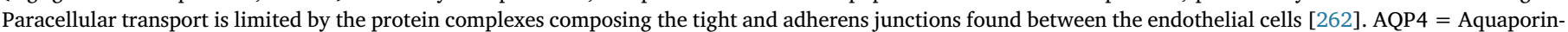

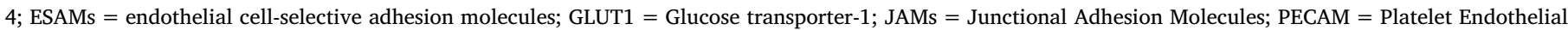
Cell Adhesion Molecule; VE-Cadherin = Vascular Endothelial Cadherin; $\mathrm{ZO}=$ Zonula Occludens.

the BBB is largely seen as an obstacle in getting therapeutic compounds to their targets in the brain. Getting across the BBB is not a trivial matter, the BBB's structure actively works against pharmacological interventions and it is estimated that approximately $98 \%$ of drugs do not cross the BBB [45]. Typically, drugs that are negatively charged and larger than approximately $400 \mathrm{Da}$ will not cross the BBB on their own and larger molecules (even if positively charged) require a transporter to carry them across the BBB $[46,47]$. As such, while novel therapeutics may be developed that show good clinical efficacy, they more often than not fail to pass the BBB except at very high doses and thus with greater systemic off-target side effects [48]. Numerous methods have been developed to aid with delivery of drugs to the CNS including intranasal delivery [49], the use of nanomaterials [50], RNA interference [51], and focused ultrasound [52]. However, the majority of approaches assume a "normal" BBB is present in patients and often do not take into account either the dynamic nature of the BBB [6] or that the BBB may be dysfunctional in psychiatric populations.

\section{Associations between the BBB and neuropsychiatric disorders}

Given the extent that the cerebral vasculature permeates the brain, alterations in blood vessel physiology may present a parsimonious mechanism that can explain the complex and variable presentation of psychiatric disorders. Disruption of the vasculature (not necessarily limited to changes in BBB permeability) could affect multiple regions and systems simultaneously, thus impacting on several cognitive and behavioural functions at the same time. Recently, close examination of the integrity of the $\mathrm{BBB}$ in various disorders has revealed that $\mathrm{BBB}$ permeability is altered in many neurological conditions and focal changes in the BBB have been implicated in TBI and epilepsy. The case for BBB disruption in TBI is strong [53-55], with a biphasic opening of the BBB believed to be part of the symptoms underlying the neurological effects observed following TBI [56]. Similarly, the dynamism of the $\mathrm{BBB}$ appears to be related to changes in neuronal firing during epilepsy [57-60].

In addition to these neurological disorders, there is increasing evidence that the $\mathrm{BBB}$ is altered in a more diffuse manner in many 


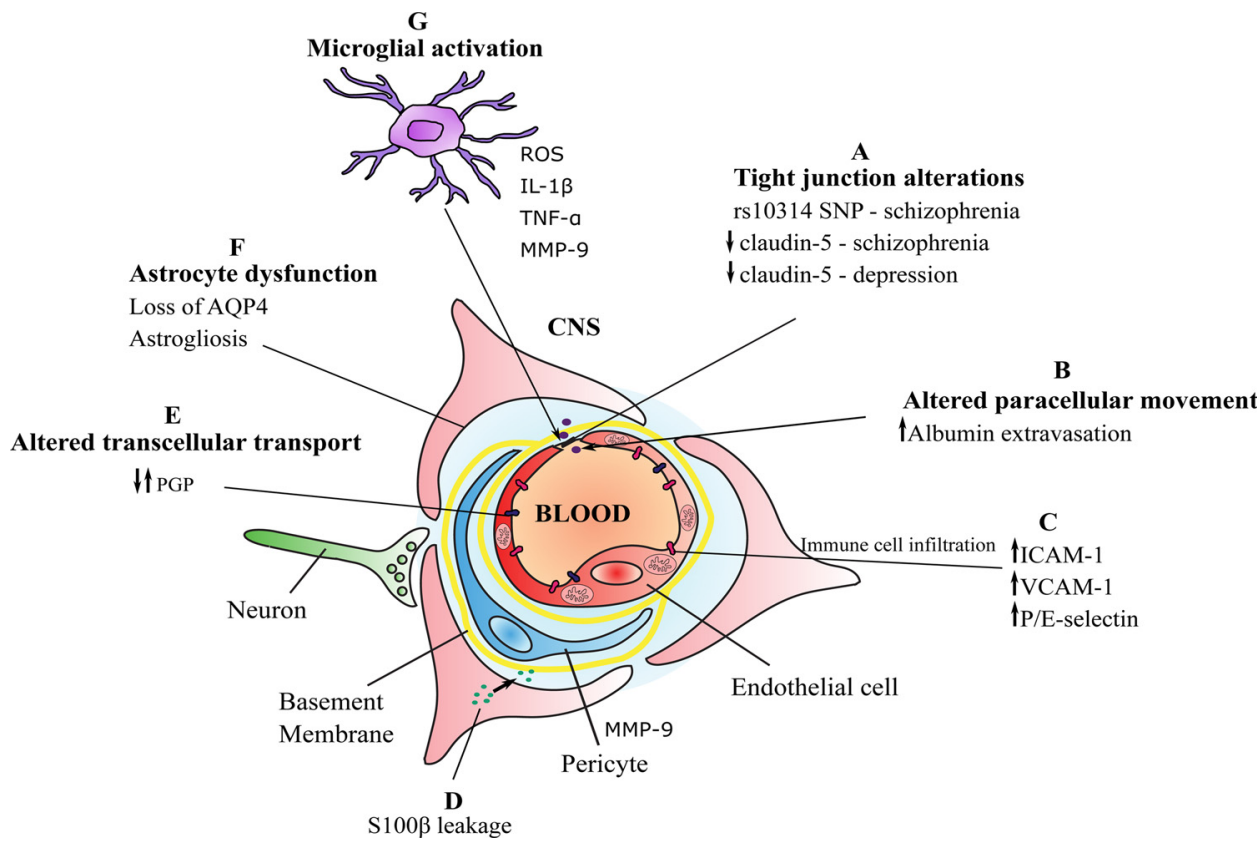

Fig. 2. Schematic summary of experimental data of structural and functional BBB alterations in psychiatric disorders. (A) Alterations of tight junction proteins (such as claudin- 5 gene polymorphisms) mediate increased paracellular movement of molecules. (B) Increased paracellular movement of macromolecules such as albumin increase CSF:serum albumin ratio. (C) Increased expression of cell adhesion molecules facilitates CNS entry of leukocytes and peripheral cytokines via the paracellular and transcellular pathways. (D) Secretion of astrocytic proteins such as $S 100 \beta$ from damaged astrocytes, cross a leaky BBB and are detectable in the blood. (E) Variability in the expression and function of transcellular transporters such as PGP may affect the brain concentration of psychiatric drugs and could contribute to treatment resistance in some patients. (F) Dysfunction of astrocytes and other glial cells may contribute to dysfunction of the neurovascular unit. (G) Microglial activation results in the production of reactive oxygen species and cytokines that act on the BBB to further exacerbate BBB permeability via alterations to adherens junctions and tight junctions. Increased production of matrix metalloproteinases from microglia and pericytes results in degradation of the basement membrane and junctional complexes, increasing BBB permeability [263].

psychiatric disorders including schizophrenia, autism spectrum disorders, and mood disorders. Here, we present the clinical and preclinical evidence for BBB dysfunction in such psychiatric conditions, highlighting changes in the endothelial tight junction in particular (Fig. 2).

\subsection{Schizophrenia}

Schizophrenia is a neurodevelopmental disorder that manifests as a mix of what are designated as positive and negative symptoms along with associated cognitive impairments, particularly in attention and memory [61,62]. There is accumulating evidence suggesting that anomalies of the microvasculature are involved in the pathogenesis of schizophrenia $[63,64]$. Up to $50 \%$ of deaths of individuals with schizophrenia are accounted for by cardiovascular disease [65]. Indeed, schizophrenic patients have a significantly increased burden of cardiovascular disease compared to the general population and as such, measurements of endothelial dysfunction may prove beneficial in identifying high-risk individuals. Identifying markers of vascular endothelial dysfunction may offer alternative approaches to identifying at risk individuals.

\subsubsection{Post-mortem studies in schizophrenia}

Several early studies focussed on post-mortem analysis of brain capillaries from control and schizophrenia individuals. Post-mortem studies have often been limited by small sample sizes, poorly matched controls, confounding effects of medications, storage conditions and preparation of brain tissue that may potentially introduce detrimental effects. Morphological differences in capillaries and NVU cell types have been observed in the prefrontal and visual cortex of schizophrenia patients along with vacuolar degeneration of endothelial cells, astrocyte-foot processes and thickening of the basement membrane [66]. In a follow-up study, reductions of capillary density were found to associate with negative symptoms of schizophrenia [67]. Structural abnormalities have also been detected in the brains of schizophrenic patients treated with anti-psychotic drugs including reduced capillary diameter, extracellular matrix deposition and perivascular edema but also pinocytosis and vacuolization [68]. However, this study involved a sample size of just three patients who were treated with anti-psychotics.
Several studies have shown abnormalities in other NVU components, notably astrocytes. Reductions in the number of pericapillary oligodendrocytes in the prefrontal cortex [69] and decreased numbers of perivascular glial fibrillary acidic protein (GFAP) positive astrocytes in the prefrontal cortex [70] and anterior cingulate cortex [71] was observed in schizophrenia patients.

\subsubsection{Neuroimaging studies in schizophrenia}

Gross anatomical changes in brain structure in vivo have also been observed with deficits in grey matter volume primarily in cortical brain regions compared to unaffected controls [72]. Neuroimaging studies have identified consistent structural abnormalities in schizophrenic patients. Volume reductions in the medial temporal lobe (memory), left posterior superior temporal gyrus (auditory processing and language) [73] as well as ventricular enlargement have been consistently observed [74]. Neuroimaging studies have not assessed BBB changes in schizophrenia patients with the few early studies confounded by small sample size, difficult interpretation of results and imprecise techniques. Advancements in dynamic contrast-enhanced-magnetic resonance imaging (DCE-MRI) for quantitative assessment of BBB permeability may be useful for detecting subtle BBB abnormalities [75], but these have yet to be applied to psychiatric patients. A recent MRI study using a 7T scanner reported alterations in the volume of small arterial and arteriolar cerebral vessels in cerebral vessels throughout the brain, suggesting that microvascular anomalies may be widespread across the brain [76] and these could partly explain the lower grey matter volumes associated with schizophrenia. This work built on previous findings of aberrant $\mathrm{CBF}$ and cerebral blood volume associated with schizophrenia [77]. As these studies focussed on smaller vessels, it has relevance to the $\mathrm{BBB}$. More recently, the CNS endothelium has been observed to more intimately regulate $\mathrm{CBF}$ through activation of the $\mathrm{K}_{\mathrm{IR}} 2.1$ inward rectifier $\mathrm{K}^{+}$channel to produce a rapidly propagating retrograde hyperpolarization that causes upstream arteriolar dilation, resulting in increased blood flow to the capillary bed. Initially this was performed ex vivo in brain slices via the addition of $\mathrm{K}^{+}$and subsequently repeated in vivo via addition of $\mathrm{K}^{+}$adjacent to a post arteriolar capillary which produced a rapid increase in red blood cell flux when measured by two-photon laser-scanning microscopy [78]. It will be interesting to determine if dysfunction of this process is evident in 
schizophrenia as alterations of CBF have been observed across several brain regions in schizophrenia with reduced CBF being associated with negative symptoms of schizophrenia notably in the frontal lobe [79-81].

Glutamate is the primary excitatory neurotransmitter in the brain and disturbances in glutamate-dependent neurotransmission have been documented in numerous psychiatric disorders including schizophrenia. This hypothesis is based on the findings that $N$-methyl-D-aspartate (NMDA) receptor antagonists can induce schizophrenia-like symptoms [82]. A meta-analysis of glutamate levels by magnetic resonance spectroscopy revealed elevations in glutamate and glutamine levels in the basal ganglia and medial temporal lobe [83]. As glutamate modulates BBB permeability [84], regional disturbances in glutamate levels may alter regional BBB permeability. Additionally, abnormal glutamate homeostasis at the BBB may contribute to psychopathology. Glutamate is actively transported out of the brain by amino acid transporters at the BBB to maintain interstitial fluid concentrations of glutamate at a fraction of the blood [85-87]. Dysfunctional astroglial glutamate transporter expression has been observed in schizophrenia [88]. In addition, polymorphisms of these transporters have been associated with cognitive dysfunction in schizophrenia patients [89].

\subsubsection{Markers of $B B B$ permeability in schizophrenia}

Increased BBB permeability has been proposed as a potential risk factor in schizophrenia, though whether it is a primary cause or a related symptom is unclear [63]. The gold-standard technique for measuring BBB permeability in humans is measurement of the cerebrospinal fluid (CSF):serum albumin ratio (QAlb). This test compares the concentration of albumin in the blood compared to the CSF. Albumin is typically present at concentrations approximately 200 times lower than blood, therefore, an increased QAlb suggests that increased quantities of albumin have been able to pass from the blood into the CSF due to an impaired barrier. This test has been used to detect BBB dysfunction in several psychiatric studies [90]. In a study of 63 psychiatric subjects and 4100 controls, a subset of psychiatric patients (14 major depressive disorder and bipolar disorder and 14 schizophrenia) had CSF abnormalities reflecting BBB dysfunction. BBB dysfunction was represented as increased serum albumin levels with BBB dysfunction being the only sign of dysfunction in $24 \%$ of cases [91]. A dysfunctional blood-CSF barrier has also been reported in patients with several forms of dementia including Alzheimer's disease and frontotemporal dementia with elevated QAlb [92]. However, analysis of QAlb has its limitations as increased QAlb can result from other factors including low rates of CSF production, increased subarachnoid flow resistance or blocking of arachnoid villi causing reduced outflow into venous. Additional problems stem from the small sample sizes of the studies and the confounding factor of anti-psychotic medication [91].

The calcium-binding peptide $S 100 \beta$ is produced mainly by astrocytes and is abundantly expressed by neurons in the brain. In healthy individuals, $S 100 \beta$ is almost undetectable in the serum. Increased serum concentrations of $S 100 \beta$ have therefore been used to associate CNS pathology with BBB dysfunction. There is accumulating evidence showing increased levels of S100 $\beta$ in the blood, CSF and brains of schizophrenic patients [93-95]. Additionally, plasma S100 $\beta$ levels were positively associated with the negative symptoms $[96,97]$ and cognitive impairments [98] associated with schizophrenia. However, whether this increase in $S 100 \beta$ is directly reflecting increases in BBB permeability or merely increased production and/or secretion by glial cells or degeneration of glial cells [99] has yet to be elucidated. Additionally, S100 $\beta$ has been found to be secreted by adipose tissue outside the CNS [100] calling into question the interpretation of the results and validity of $S 100 \beta$ as a pure marker of BBB permeability.

A limited number of studies have examined blood concentrations of BBB components. Vascular endothelial dysfunction has been suggested in several studies with increased peripheral concentrations of endothelial cell adhesion molecules such as soluble P-selectin and L- selectin in the serum and plasma of untreated acute schizophrenic patients compared to controls [101,102] and alterations in the levels of vascular endothelial growth factor (VEGF), intercellular adhesion molecule 1 (ICAM-1), and vascular cell adhesion molecule 1 (VCAM-1) in individuals with schizophrenia [103]. A recent study identified elevated serum levels of VEGF in schizophrenia patients compared to controls that were associated with structural abnormalities in the prefrontal cortex [104]. However, peripheral VEGF levels appear to be low in schizophrenics who are not being treated with antipsychotics, with VEGF levels only increasing above normal levels following antipsychotic treatment [105]. Central levels of VEGF in schizophrenic patients have been shown to be lower than those of normal controls [106]. Taken together, these studies suggest the possibility of increased endothelial cell activation in the cerebral vasculature of individuals with schizophrenia. Furthermore, atypical antipsychotics such as risperidone contributed to vascular dysfunction in diabetic rats via activation of endothelial cell adhesion molecules such as ICAM-1, VCAM-1 and soluble L-selectin [107].

\subsubsection{The $B B B$ and immune response in schizophrenia}

Activation of cell adhesion molecules on the vascular endothelium may contribute to increased transendothelial migration of lymphocytes and monocytes. Matrix metalloproteinase-9 (MMP-9) is a $92 \mathrm{kDa}$ protein that belongs to the family of zinc and calcium dependent endopeptidases. Recently, it has been shown to negatively affect CNS disorders such as epilepsy and TBI but few studies have investigated MMP levels in schizophrenia. Two studies reported increased concentrations of MMP-9 and tissue inhibitors of metalloproteinase (TIMP) in schizophrenia [108,109], although another study found no differences between patients and controls from a total of 63 patients with chronic schizophrenia [110]. Central and peripheral inflammation have both been identified in schizophrenia [111] and given that inflammation is known to modify the BBB directly $[112,113]$ and to impact on brain function in general [114], it is perhaps unsurprising that changes in expression of endothelial specific genes have been investigated and these have implicated the role of the BBB in the immune response in schizophrenia. This may be occurring over the lifetime of an individual, including during early development where maternal stress and inflammation could interfere with BBB development [115]. Microarray analysis of endothelial cells isolated by laser capture microdissection from post-mortem schizophrenic patients and healthy controls found downregulation of endothelial cell genes involved in ion transport, cell proliferation and adhesion, suggesting a dysfunction of the BBB [116]. As the BBB enforces the "immune privileged" status of the brain, changes in BBB components such as cell adhesion molecules may increase peripheral immune cell infiltration to the brain. Previously, this has been shown to correlate with cognitive and behavioural changes in animal models in response to systemic inflammation [117]. Further evidence linking BBB disruption and immune responses was reported by Hwang and colleagues who used RNA-seq data from the hippocampus of control and schizophrenia subjects that identified 144 genes differentially regulated in schizophrenia cases compared to unaffected controls, the majority of which are involved in the immune/inflammation response. Additionally, most of these differentially expressed immune system genes were more likely to be expressed in endothelial cells of the BBB, blood monocytes within blood vessels and perivascular astrocytes than in lymphocytes or microglia [118]. Another study identified 23 genes up-regulated in the choroid plexus of 29 schizophrenia subjects compared to 26 unaffected controls related to biological processes involved in immune responses and amino acid transport. The differential expression of these genes positively correlated with the amounts of inflammatory proteins in the serum and frontal cortex including c-reactive protein, cortisol, MMP-9 and TIMP-1 [119].

Recent interrogation of microarray datasets from 15 cerebrocortical regions and the hippocampus of individuals with schizophrenia identified 657 differentially regulated genes, 311 of which correspond to a 
subset uniquely enriched in endothelial cells. Most of these endothelial cell-enriched genes that were downregulated in schizophrenia are involved in angiogenesis pathways [120]. Another gene expression study examining VEGF mRNA from the dorsolateral prefrontal cortex of 16 individuals with schizophrenia and 18 psychiatrically normal controls found significant decreases in VEGF in the schizophrenia group [106]. Cerebral vascularisation is mediated by VEGF and VEGF significantly contributes to angiogenesis by stimulating neovascularisation and is intimately involved in the regulation of CBF. Additionally, VEGF has a key role in neurophysiology as suppression of neural VEGFR2 impairs hippocampal-dependent synaptic plasticity and long-term potentiation and consolidation of emotional memory in mice [121]. Studies specifically focussing on other BBB molecular components such as tight junction proteins have been limited.

\subsubsection{Tight junction dysfunction in schizophrenia}

Transcriptomic analysis of the prefrontal cortex have pointed to the endothelial tight junction signalling as an area of interest in schizophrenia with 12 out of 21 tight junction-related genes analysed showing a reduction in schizophrenia patients compared to controls [122]. Expression of claudin- 5 mRNA in the prefrontal cortex (but not the visual cortex) is significantly elevated in schizophrenia patients and this is accompanied by a decrease in claudin- 5 protein [123] (Table 1). It has been observed in a number of populations that there is a weak genetic association between claudin- 5 and the development of schizophrenia, in particular the allele rs10314 has been singled out as being a potential risk factor for psychosis [124-128] but this may be population-specific as the association was not replicated in a Japanese population [129]. rs10314 is a single nucleotide polymorphism (SNP) that produces only about half of the amount of protein as the wild type claudin- 5 gene [57], meaning that individuals with that allele may be producing less claudin-5 than is typical. The effect of this allele is particularly striking in 22q11 deletion syndrome (22q11DS). In 22q11DS, there is a vastly increased risk of developing schizophrenia compared to the normal population (as high as $30 \%$ in 22q11DS [130];). Interestingly, claudin-5 lies within the deleted region in 22q11DS, meaning that individuals are haploinsufficient for claudin-5 and therefore only producing $50 \%$ as much claudin- 5 compared to the normal population. Recently, we have shown that there is a significant association between the rs10314 variant and a diagnosis of schizophrenia in 22q11DS [57], suggesting that

\section{Table 1}

Summary of findings from ${ }^{1}$ Fiorentino et al. [154], Greene et al. [57], and Nishiura et al. [123]. Greene and colleagues specifically looked at ${ }^{2}$ prefrontal cortex, and Nishiura and colleagues looked at both ${ }^{3}$ prefrontal cortex and ${ }^{4}$ visual cortex. Arrows indicate a significant increase or decrease in mRNA or protein levels, $n s$ denotes no significant difference between schizophrenia and controls, - denotes that this measurement was not performed.

\begin{tabular}{|c|c|c|}
\hline Tight Junction Component & Cortex & Cerebellum \\
\hline \multicolumn{3}{|l|}{ Claudin-1 } \\
\hline mRNA & - & - \\
\hline Protein & - & - \\
\hline \multicolumn{3}{|l|}{ Claudin-3 } \\
\hline mRNA & $\mathrm{ns}^{1}$ & - \\
\hline Protein & - & - \\
\hline \multicolumn{3}{|l|}{ Claudin-5 } \\
\hline mRNA & $\mathrm{ns}^{1} / \uparrow^{3} / \mathrm{ns}^{4}$ & $\mathrm{~ns}^{1}$ \\
\hline Protein & $\mathrm{ns}^{1} / \mathrm{ns}^{2} / \downarrow^{3} / \mathrm{ns}^{4}$ & $\mathrm{~ns}^{1}$ \\
\hline \multicolumn{3}{|l|}{ Claudin-12 } \\
\hline mRNA & $\mathrm{ns}^{1}$ & $\mathrm{~ns}^{1}$ \\
\hline Protein & $\mathrm{ns}^{1}$ & $\mathrm{~ns}^{1}$ \\
\hline \multicolumn{3}{|l|}{ Occludin } \\
\hline mRNA & - & - \\
\hline Protein & - & - \\
\hline \multicolumn{3}{|l|}{ Tricellulin } \\
\hline mRNA & $\mathrm{ns}^{1}$ & ns \\
\hline Protein & - & - \\
\hline
\end{tabular}

these individuals are producing only about $25 \%$ as much claudin- 5 compared to the normal population and therefore may have greater BBB permeability.

Support for the role of claudin-5 in schizophrenia also comes from preclinical work, where antipsychotic drugs have been shown to directly increase claudin-5 expression in vitro and in vivo [57]. A constitutive claudin- 5 knockout mouse has been developed but these mice die within hours of birth [131], making them unsuitable for understanding neurodevelopmental disorders such as schizophrenia. Recently, we developed and characterised an inducible knock-down mouse model allowing for long-term suppression of claudin-5 in adult mice [57]. In this model, we showed that claudin-5 suppression was associated with a number of behavioural impairments that are suggestive of psychosis-like symptoms including impairments in learning and memory, increases in anxiety, and impairments in sensorimotor gating. However, such extensive and lengthy suppression of claudin-5 has implications beyond psychosis as the mice eventually developed seizures and died.

\subsection{Autism spectrum disorders}

Autism spectrum disorders (ASD) are a class of neurodevelopmental disorders associated with problems relating to communication and social interaction, repetitive behaviours, anxiety, and cognitive impairments [132-134]. As in schizophrenia, there is mounting evidence that there may be a vascular component to this category of disorders. Changes in grey matter that have been observed in ASD have been suggested to reflect changes at multiple components of the NVU including dendritic density, glial cell numbers and morphology, along with changes in the vasculature [135]. Work on the ASD transcriptome has identified dysregulation of vascular development in autism (in addition to other processes including regulation of the synapse and inflammation) [136]. Indeed, post-mortem analysis of brain tissue from individuals with ASD showed significantly higher levels of markers associated with endothelial cells and pericytes, along with increases in angiogenesis and blood vessel plasticity compared to controls [137]. In Fragile X syndrome, approximately one third of individuals show ASDlike symptoms [138] and there is evidence to suggest that the presence of abnormalities in the cerebral vasculature in this population [139]. Additionally, in a Fragile X mouse model, there is an increase in blood vessel density in middle-aged mice [140,141].

\subsubsection{Markers of $B B B$ permeability in $A S D$}

There have been few studies directly examining the properties of the $\mathrm{BBB}$ in ASD but a number of studies have recently pointed to alterations in BBB function in these conditions. Genetic screening has identified multiple barrier-related functions for known autism-associated genes, indicating that some autism-associated genes are regulating the BBB to some degree [142]. Soluble forms of endothelial cell markers such as PECAM are lower in children and adults with ASD [143-145]. A subset of children with ASD showed significantly higher levels of autoantibodies against brain endothelial cells in their sera compared to neurotypical controls ( $27 \%$ versus $2 \%$ respectively), indicating that there may be increased permeability of the BBB in some ASD individuals [146]. The evidence for whether BBB disruption is present in ASD based on molecular markers of increased BBB permeability such as S100B, GFAP, neuron-specific enolase (NSE), myelin basic protein (MBP), and creatine kinase brain isoenzyme (CK-BB) is mixed. One recent study found that S100B, GFAP, and NSE did not differ between ASD and control children but did find that GFAP levels were significantly higher in ASD individuals and GFAP levels positively correlated with scores on the Childhood Autism Rating Scale [147]. Other studies did find significantly increased levels of $S 100 \beta$ in serum [148] and in plasma [149] for children with ASD and that this correlated with increased levels of the cytokine tumour necrosis factor-alpha (TNF- $\alpha$ ) [149]. In a neonatal population, there were significantly higher levels 
of NSE and CK-BB in the ASD group compared to controls [150]. Lv and colleagues also found that within the ASD group, the levels for both these brain-specific molecules were significantly higher in low-functioning ASD individuals compared to high-functioning ASD individuals. However, there were no correlations between scores on the Neonatal Behavioral Assessment Scale and levels of these molecules. Given that the neonatal $\mathrm{BBB}$ has not yet fully formed, it is impossible to say whether these findings relate to changes at the level of the BBB in ASD at this point of development. Animal models of ASD have given some support for increased BBB permeability in ASD; in the valproic acid model of autism in rats, increases in BBB permeability (measured using Evan's Blue) have been reported [151-153] that can be ameliorated with treatment with the NMDA receptor agonist memantine [151], the antibiotic minocycline [152], or the melatonin receptor agonist agomelatine [153].

\subsubsection{Structural changes at the $B B B$ in $A S D$}

Further evidence for differences in the BBB in ASD comes from direct examination of the $\mathrm{BBB}$ itself. The endothelial adhesion molecules platelet endothelial adhesion molecule-1 and P-selectin have been shown to be downregulated in children with ASD, suggestive of impaired transport of leukocytes across the BBB in ASD [144]. In postmortem brain tissue samples, there are a number of changes in the tight junction proteins that are suggestive of an altered BBB in the cortex and the cerebellum (Table 2) [154]. Interestingly, Fiorentino and colleagues also showed that there were disruptions in at least one tight junctionrelated protein (occludin or claudin-1) in $75 \%$ of biopsies made in the small intestine but these findings did not reach significance overall. They did find that pore-forming proteins (claudin-2, -10, and -15) were significantly increased in ASD small intestine, reflecting a more permeable intestinal barrier [154]. This relationship between neurological and gastrointestinal barrier disruption in ASD remains an important area for future research given the high levels of gastrointestinal problems associated with ASD [155] and the proposed interactions between the gut microbiome, inflammation, and neurological symptoms of ASD [156-159].

Similarly, changes in transcellular transport mechanisms have also been identified in ASD, particularly the transport of amino acids [160] and folate [161] into the brain. Altered amino acid profiles in individuals with autism, epilepsy, and intellectual disability is associated with impaired transport of branch chain amino acids across the BBB [162] and animal models blocking the transcellular transport of branch chain amino acids into the brain results in the development of an ASD-

\section{Table 2}

Summary of findings from Fiorentino et al. [154]. Arrows indicate a significant increase or decrease, $n s$ denotes no significant difference between ASD and controls, - denotes that this measurement was not performed.

\begin{tabular}{|c|c|c|c|}
\hline Tight Junction Component & Cortex & Cerebellum & Intestine \\
\hline \multicolumn{4}{|l|}{ Claudin-1 } \\
\hline mRNA & ns & ns & ns \\
\hline Protein & - & - & - \\
\hline \multicolumn{4}{|l|}{ Claudin-3 } \\
\hline mRNA & $\uparrow$ & ns & - \\
\hline Protein & - & - & - \\
\hline \multicolumn{4}{|l|}{ Claudin-5 } \\
\hline mRNA & $\uparrow$ & $\uparrow$ & - \\
\hline Protein & $\uparrow$ & $\uparrow$ & - \\
\hline \multicolumn{4}{|l|}{ Claudin-12 } \\
\hline mRNA & $\uparrow$ & $\uparrow$ & - \\
\hline Protein & $\downarrow$ & ns & - \\
\hline \multicolumn{4}{|l|}{ Occludin } \\
\hline mRNA & ns & ns & ns \\
\hline Protein & - & - & - \\
\hline \multicolumn{4}{|l|}{ Tricellulin } \\
\hline mRNA & $\uparrow$ & ns & ns \\
\hline Protein & - & - & - \\
\hline
\end{tabular}

like phenotype in these mice [160]. The presence of folate receptor autoantibodies (FRAs) in some children with ASD inhibit the transport of folate (in the form of methylfolate) from the periphery into the brain, indicated by significantly lower levels of 5-methyltetrahydrofolate in the cerebrospinal fluid of children with ASD who are positive for FRAs [163]. FRAs have also been identified in individuals with Rett syndrome [164] and infantile low-functioning autism with neurological abnormalities [165]. Treatment in leucovorin (which is used to treat folate deficiency) resulted in an improvement in a number of scores relating to ASD symptomology $[163,165]$. The exact interactions between FRAs and ASD development is not yet clear but measurements of behaviour, inflammation, and levels of serum B12 appear to be different for individuals with the binding versus those with the blocking type of FRA [166].

\subsubsection{The $B B B$ and immune response in $A S D$}

As in schizophrenia, changes in immune function have also been identified in individuals with ASD [167], suggesting that systemic inflammation may be affecting the brain due to an impaired BBB in individuals with ASD $[115,168]$. Seizures in ASD have been proposed to be due to allergic-, inflammation- or stress-induced disruption of mast cells leading to increased BBB permeability [169], a mechanism that has been suggested to underlie ASD symptoms more broadly [170-173]. Modulation of the BBB by MMP-9 has been suggested in ASD [174] as high amniotic fluid levels of MMP-9 have been linked to the development of ASD [175] and Mmp-9 mRNA is significantly higher in the cortex of individuals with ASD [154] and with Fragile X syndrome [176]. In rodent models of ASD and Fragile X, pharmacologically-induced decreases in MMP-9 have been shown to associate with improvements in ASD-like behaviours in these animals [177-180]. Similarly, Fmr1 and Mmp-9 double knock-out mice show less of the ASDlike symptoms than the Fmr1 single knock-out mouse model of Fragile X [176].

\subsection{Mood disorders}

Mood disorders (including depression, anxiety, and bipolar disorder) affect approximately $20 \%$ of the population based on lifetime prevalence [95]. Major depressive disorder (MDD) is the most prevalent mood disorder and a leading cause of worldwide disability [181]. Individuals with depression are at increased risk for cardiac morbidity and mortality with depression being more frequently observed in patients who suffer from cardiovascular disorders [182]; individuals who have suffered a myocardial infarction are three times more likely to develop depression [183]. While oxidative stress and neuroinflammation are implicated in the neurobiology of depression [64], recent evidence has associated BBB and NVU dysfunction with depression. NVU dysfunction has also been implicated in the aetiology of MDD with deficits in glial cell density and function evident in human post-mortem brain studies [184-186]. These findings have also been supported in various animal studies of depression [185-188]. In addition, microglial activation has been reported in the subgenual anterior cingulate cortex [189] further reinforcing the immunological link to MDD.

\subsubsection{Markers of $B B B$ permeability in mood disorders}

Evidence for the involvement of BBB dysfunction in depression have mainly been derived from studies of the CSF to serum ratio of various molecules. A subset of individuals with MDD with elevated QAlb ratios suggests an increased permeability of the BBB and/or blood-CSF barrier. Adult women with MDD had higher CSF concentrations of amyloid beta as well as an increased QAlb [190]. Additionally, a clinical study found increased CSF to serum levels of peripheral markers including albumin and urate in depressed patients, suggestive of a compromised BBB. Furthermore, increased QAlb ratios were associated with a slowing of the electroencephalogram (EEG, a measure of brain dysfunction) and suicidality [191]. In another study, 9 out of 24 
individuals with an affective spectrum disorder (controls $n=4100$ ) had an increased QAlb [91]. Post-mortem analysis of brain tissue has shown that there is downregulation of the tight junction protein claudin-5 in the nucleus accumbens of patients with depression [192]. Suppression of claudin- 5 has also been shown to induce behaviours associated with depression and anxiety in mice including deficits in social behaviour, grooming, and increased anxiety [57].

Like albumin, serum levels of S100 $\beta$ have been used to assess BBB dysfunction in depressed individuals. Increased levels of $S 100 \beta$ have also been reported in patients with acute major depression and during manic episodes. S100 $\beta$ levels can be attenuated by antidepressant treatment [193]. The effect of antidepressant treatment on S100 $\beta$ levels correlated positively with clinical improvement in patients [194]. Additionally, S100 $\beta$ levels seem to be higher in patients with MDD compared to those with bipolar disorder [195] and older patients with MDD have higher levels of $S 100 \beta$ compared to younger patients [196]. In a clinical trial comparing coronary artery bypass grafting procedures, changes in levels of $S 100 \beta$ positively correlated with changes in depressive symptom severity [197]. In rodent studies, overexpression of $\mathrm{S} 100 \mathrm{\beta}$ in female mice induces depression-like behaviour in the forced swim task [198].

\subsubsection{The $B B B$ and immune response in mood disorders}

While numerous studies have identified potential dysfunction of the $\mathrm{BBB}$ in depression, there is a paucity of studies investigating the potential role of $\mathrm{BBB}$ dysfunction in the pathophysiology of depression. A possible consequence of BBB dysfunction in depressive-like behaviour has been linked to the brain penetration of circulating pro-inflammatory cytokines [199-201]. Animal models of depression have provided insight on the role of the BBB in depression. In a stress-induced model of depression, stress was associated with reduced expression of claudin-5 and occludin and neurovascular dysfunction [202]. Similarly, stress induced increases in the permeability of the BBB to intravenously injected tracers [200] and to Evans blue [203]. However, the link between stress and BBB dysfunction is controversial [204]. Recently, the role of claudin-5 in depression was evaluated in the chronic social defeat stress model, a mouse model of depression. In this model, loss of claudin-5 in the nucleus accumbens was associated with increased depression-like behaviours and infiltration of the peripheral cytokine interleukin-6 (IL-6). This behavioural phenotype could be rescued following chronic anti-depressant treatment [192].

Systemic inflammation is known to impact directly on depression, suggesting that the $\mathrm{BBB}$ may be a mediating factor in the development of depression [205]. Within the CNS, microglia are the resident immune cell that are involved in phagocytosing cellular debris and synapses. Following trauma, microglia actively migrate towards the site of damage, such as ischemia and neurodegeneration, and engulf and eliminate neuronal cellular debris following cell death. However, prolonged activation of microglia is a hallmark of neuroinflammation. Activated microglia can impair the BBB via secretion of reactive oxygen species [206] and cytokines including tumour necrosis factor $\alpha$ and IL-1 $\beta$ [207-209] As such, microglia dysfunction has been implicated in depression [210]. Deficits in glial cell density, coverage of blood vessels and function are evident in human post-mortem brain studies, linking NVU dysfunction in the aetiology of MDD [184-186,211]. Astrocyte dysfunction has been consistently observed in MDD with reduced density and coverage and loss of the water channel transporter aquaporin 4 (AQP4) [186,211,212]. These findings have also been supported in various animal studies of depression [185-188]. In addition, microglial activation has been reported in the subgenual anterior cingulate cortex [189] further reinforcing the immune link to MDD. Furthermore, levels of the cell adhesion molecule ICAM-1 are increased in depressed patients in the prefrontal cortex [213,214]. ICAM-1 is involved in leukocyte infiltration to the brain and selective serotonin reuptake inhibitors can reduce the expression of adhesion molecules to potentially mediate anti-inflammatory effects [215]. These studies highlight the critical need for homeostasis of the neuronal microenvironment to regulate leukocyte interactions with the brain and how alterations of these processes can lead to BBB hyperpermeability, neuroinflammation and neuronal degeneration.

\subsubsection{Therapeutic considerations in mood disorders}

The main treatment for depression is anti-depressant medication; however, up to two thirds of individuals will not respond to the first prescribed antidepressant and up to $33 \%$ will not respond to multiple interventions [216]. At the BBB, P-glycoprotein (PGP) is actively involved in extruding toxins and xenobiotics out of the brain. PGP is critically involved in drug absorption and disposition. PGP has broad substrate specificity and actively effluxes larger molecular weight hydrophobic drugs out of the brain [217]. Endothelial cells of the BBB restrict the entry of amphiphilic compounds such as anti-depressants. As anti-depressants must cross the BBB to act on the brain, differences in the functionality of PGP may lead to variable brain concentrations of anti-depressants and subsequent variability in therapeutic response. Furthermore, many anti-depressants are PGP substrates [218] with PGP knockout mice having significantly higher brain concentrations of many anti-depressants [219]. It can be hypothesised that genetic variants in the PGP gene influence PGP expression at the BBB with higher expression extruding a greater quantity of anti-depressants and reduced PGP expression facilitating entry of anti-depressant drugs into the CNS. Previously, a significant association has been reported between a SNP in the gene encoding PGP, (ATP-binding cassette, sub-family B member 1 ), and MDD [220]. Meta-analysis of 16 pharmacogenetic studies on PGP polymorphisms identified the rs2032583 SNP that was nominally associated $(\mathrm{P}=0.035)$ with anti-depressant treatment outcome [221]. MDD patients undergoing anti-depressant treatment had significantly increased PGP function as measured by positron emission tomography [222]. Similarly, the brain uptake of L-5-hydroxytryptophan, the precursor of serotonin, is reduced in depressed individuals [223]. In animal studies, mice deficient for PGP displayed increased depression-like behaviours including social withdrawal, concomitant with increased microglia cell density in the hippocampus [224]. In animal models of depression, social stress induced reductions in PGP function in rats subjected to chronic stress as assessed by the uptake of verapamil, while chronic anti-depressant administration exerted the opposite effect [225]. Furthermore, inhibition of PGP increased brain concentrations of the anti-depressant imipramine [226].

\section{The BBB in clinical practice}

As noted above, the extensive relationship between the cerebral vasculature and the brain may explain how small changes in vascular function could impact on numerous neuronal networks and systems. However, we do not expect all patients with schizophrenia, ASD, or an affective disorder to show the same characteristics at the vascular level and it is likely that many different changes in vascular or BBB function can all manifest in similar symptoms (i.e. a change in tight junction permeability and altered levels of AQP4 could both change the environment of the cerebral parenchyma to cause similar disruption of neuronal activity). The links between BBB (and vascular function in general) and psychiatric disorders is in its infancy and much work needs to be done to settle whether the dysfunction seen in the BBB in these disorders are causative or whether they are in fact symptoms arising from other changes in the brain.

In addressing these open avenues of enquiry, we review some of the clinical implications for BBB dysfunction in psychiatry and whether BBB dysfunction can be used as part of diagnosis (or refinement of diagnosis, e.g. identification of drug-resistant populations) or in understanding the physiological pathologies in the various types of psychiatric disorder outlined above. Should BBB dysfunction be a causative factor (or at the very least a risk factor), assessment of BBB permeability through neuroimaging and blood biomarkers, along with identification 
of risk alleles relating to BBB function, should help point clinicians towards individualised treatment plans for patients.

\subsection{Genetic markers and risk factors}

For claudin-5, a SNP (rs10314) has been weakly associated with schizophrenia but not with other psychiatric disorders (such as ASD or affect disorders) [124-129]. As highlighted in Section 3.1, claudin-5 is located in the region affected by 22q11DS resulting in individuals who are haploinsufficient for claudin-5 (along with several other genes) and are therefore more susceptible to the effect of rs10314 [57]. However, little is known about the effects of other claudin- 5 alleles which may also play a role in schizophrenia or other psychiatric disorders.

For occludin, there is no research available looking at the relationships between known variants and psychiatric disorders. It is known that the fourth transmembrane domain (TM4, located at the start of exon 4) of the OCLN gene can be present or absent in humans [227] and the TM4(-) variant may impede delivery of occludin to the tight junction in intestinal epithelial cells [228]. However, there is no data available relating to how this variant may impinge on tight junction formation in cerebral endothelial cells or how it may affect the function of the BBB.

Polymorphisms in VEGF-A have been identified as being predictive for depression [229], with rs4416670 being identified as significantly increasing the risk of developing depression [230]. Patients with MDD have higher levels of VEGF in the periphery, indicating an involvement with the development of depression even when not including VEGF genotype as a factor [231,232]. Furthermore, alterations in the VEGF signalling pathway have been implicated in patient responsiveness to antidepressants [233]. Despite VEGF being identified as being involved in schizophrenia (see Section 3.1), there is little evidence for any SNPs affecting the risk of developing schizophrenia [234].

As discussed in Section 3.3, polymorphisms in PGP have been associated with MDD [220] and the type of polymorphism is associated with the severity of MDD [235]. As this protein is important in transporting antidepressant drugs across the BBB, it is not surprising to find that polymorphisms in this gene may be predictive of whether MDD patients are likely to be responsive to particular treatments or not [235,236]. However, not all PGP polymorphisms appear to predict sensitivity to treatment type (even if they are still associated with depression) [237,238]. Meta-analysis of studies investigating the relationships between polymorphisms in PGP and depression have found that the SNP rs2032583 is the variant that shows the most promise for pharmacogenomic application [239] in depression [221]. Bearing this in mind, initial work using PGP genotyping to optimise antidepressant regimes in patients carrying rs2032583 have shown positive results [240,241].

\subsection{Dynamic contrast-enhanced-magnetic resonance imaging}

Given the evidence outlined in the previous section, it is tempting to speculate about using measurements of BBB permeability as a diagnostic measure or to perhaps target the BBB directly as a therapy. Traditional neuroimaging has identified statistical differences between normal and psychiatric populations [242] but more specialised forms of neuroimaging incorporating gadolinium as a contrast agent have provided deeper insights into how the cerebral vasculature may be altered in schizophrenia [76,77,243-246] and bipolar disorder [247].

DCE-MRI has been proven to be useful in detecting abnormal BBB permeability in oncology, with the BBB permeability determined using the pharmacokinetic parameter $\mathrm{K}^{\text {trans }}$. $\mathrm{K}^{\text {trans }}$ represents the rate by which an intravenously administered gadolinium bolus passes into the cerebral parenchyma from the bloodstream. Under normal conditions, the $\mathrm{BBB}$ highly restricts the passage of gadolinium meaning that the value of $\mathrm{K}^{\text {trans }}$ is low whereas the compromised BBB of a brain tumour [248] results in a higher $K^{\text {trans }}$ value [249]. One early study suggested that there was no BBB permeability visible during a gadolinium-enhanced MRI scan [250], but in the intervening decades there have been vast improvements in MRI technology, software, and analytical techniques. More sensitive methods for analysing the DCE-MRI signal have been developed to measure the more subtle changes in BBB permeability that occur during epilepsy [251] and TBI [252]. Although gadolinium is not typically used during clinical neuroimaging (unless something like a tumour is suspected), if BBB dysfunction is indeed a hallmark of psychiatric conditions as the evidence above suggests, then it may be useful to perform DCE-MRI in these populations. For example, not all patients with schizophrenia exhibit an altered BBB (38\% show normal claudin-5 expression [57],). Being able to identify these patients may have implications for treatment; if the BBB is dysfunctional, it may be preferable to use a drug that can normalise BBB permeability.

\subsection{Blood biomarkers of $B B B$ permeability}

As discussed in the sections above, some of the hallmark techniques for assessing a dysfunctional BBB include measurements of the serum albumin ratio and concentration of $S 100 \beta$ in the serum of patients. However these measurements have several limitations that need to be addressed before they can have potential use in a clinical setting which include: $S 100 \beta$ is also expressed by adipocytes and this source may be a confounding factor in measurements of this protein as a marker of BBB dysfunction [253]. In addition, increased $S 100 \beta$ as a marker of permeability may occur secondary to factors associated with the primary pathology of the disease such as prolonged psychiatric drug exposure, substance abuse and worsening physical health. Additionally, increased serum albumin quotients may result from a low rate of CSF production and increased subarachnoid flow resistance [254]. More recently, CSF levels of circulating tight junction proteins including claudin-5, occludin and ZO-1 have been measured and shown to correlate with BBB permeability and with damage to the $\mathrm{BBB}$ resulting from haemorrhage [255] and may hold promise in identifying BBB dysfunction in other disorders.

\subsection{Drug delivery and the $B B B$}

Much research has been devoted to modulating the BBB to remove unwanted material or to allow greater access for pharmacological agents. Several preclinical studies have highlighted the potential for RNA interference (RNAi) of tight junction proteins to reversibly modulate BBB permeability. RNAi-based suppression of claudin- 5 has been used to attenuate cerebral oedema associated with traumatic brain injury and improve neurological function in mice [256]. Additionally, RNAi-based suppression of claudin- 5 and occludin have been used in an animal model of Alzheimer's disease (AD). In the Tg2576 murine model of $\mathrm{AD}$, co-suppression of claudin- 5 and occludin selectively removed amyloid beta monomers from the brain to the blood, concomitant with improvements in cognitive function [11]. More recently, focused ultrasound with microbubbles has been employed to transiently open the BBB and deliver therapeutic molecules in numerous animal models of neurological disorders [52,257,258]. This method employs low intensity ultrasound with intravenously injected microbubbles. Once the microbubbles pass into the ultrasound field, they oscillate and mechanically disrupt the tight junctions of the BBB [259]. Recently, a phase one clinical trial of focused ultrasound began in $\mathrm{AD}$ patients to determine if this method could specifically modulate the BBB. However, if the BBB has a causal role in the development of psychiatric disorders, then therapies and strategies aimed at preventing BBB damage or repairing an already dysfunctional barrier might show promise for treating psychiatric disorders. For example, novel therapies to prevent peripheral inflammation and reduce neuroinflammation may prove beneficial in repairing the barrier. For example, high dose steroids have been used to repair the BBB in multiple sclerosis patients. Intravenous administration of methylprednisolone rapidly reduced $\mathrm{BBB}$ 
abnormalities associated with lesions [260]. In vitro studies revealed that sera from multiple sclerosis patients could downregulate tight junction proteins including claudin- 5 and occludin but this could be rescued by treatment with glucocorticosteroids [261].

\section{Conclusions}

The BBB plays a vital and nuanced role in normal brain function and the evidence is mounting for its involvement in a number of neurological disorders. In psychiatric disorders where multiple and seemingly disparate systems and functions can be affected, the role of the cerebral vasculature and BBB may be far more important than previously proposed as the entire brain may be made more vulnerable (as opposed to particular cell types or neuronal populations based on other hypotheses). However, much of the evidence in support of a vascular basis of psychiatric disorders requires deeper investigation to determine to what extent changes in BBB function may be causative in these disorders, or indeed whether these changes are as a result of earlier pathophysiological changes.

\section{Acknowledgements}

This work was supported by Science Foundation Ireland (SFI), the Health Research Board (HRB) and Bright Focus Foundation. The Campbell lab is also part of the SFI funded FutureNeuro centre.

\section{References}

[1] D.D. Clark, L. Sokoloff, et al., Circulation and energy metabolism of the brain, in: G.J. Siegel (Ed.), Basic Neurochemistry: Molecular, Cellular and Medical Aspects, Lippincott-Raven, Philadelphia, 1999, pp. 637-670.

[2] N.J. Abbott, L. Ronnback, E. Hansson, Astrocyte-endothelial interactions at the blood-brain barrier, Nat. Rev. Neurosci. 7 (1) (2006) 41-53.

[3] K.E. Schlageter, et al., Microvessel organization and structure in experimental brain tumors: microvessel populations with distinctive structural and functional properties, Microvasc. Res. 58 (3) (1999) 312-328.

[4] J. Wu, et al., 3D BrainCV: simultaneous visualization and analysis of cells and capillaries in a whole mouse brain with one-micron voxel resolution, Neuroimage 87 (2014) 199-208.

[5] C. Iadecola, The neurovascular unit coming of age: a journey through neurovascular coupling in health and disease, Neuron 96 (1) (2017) 17-42.

[6] J. Keaney, M. Campbell, The dynamic blood-brain barrier, FEBS J. 282 (21) (2015) 4067-4079.

[7] C. Greene, M. Campbell, Tight junction modulation of the blood brain barrier: CNS delivery of small molecules, Tissue Barriers 4 (1) (2016) e1138017.

[8] F. Erdo, L. Denes, E. de Lange, Age-associated physiological and pathological changes at the blood-brain barrier: a review, J. Cereb. Blood Flow Metab. 37 (1) (2017) 4-24.

[9] M.R. Halliday, et al., Accelerated pericyte degeneration and blood-brain barrier breakdown in apolipoprotein E4 carriers with Alzheimer's disease, J. Cereb. Blood Flow Metab. 36 (1) (2016) 216-227.

[10] R.D. Bell, B.V. Zlokovic, Neurovascular mechanisms and blood-brain barrier disorder in Alzheimer's disease, Acta Neuropathol. 118 (1) (2009) 103-113.

[11] J. Keaney, et al., Autoregulated paracellular clearance of amyloid-beta across the blood-brain barrier, Sci. Adv. 1 (8) (2015) e1500472.

[12] A. Salehi, J.H. Zhang, A. Obenaus, Response of the cerebral vasculature following traumatic brain injury, J. Cereb. Blood Flow Metab. 37 (7) (2017) 2320-2339.

[13] C.P. Doherty, et al., Blood-brain barrier dysfunction as a hallmark pathology in chronic traumatic encephalopathy, J. Neuropathol. Exp. Neurol. 75 (7) (2016) 656-662.

[14] V. Muoio, P.B. Persson, M.M. Sendeski, The neurovascular unit - concept review, Acta Physiol. (Oxf.) 210 (4) (2014) 790-798.

[15] N.J. Abbott, et al., Structure and function of the blood-brain barrier, Neurobiol. Dis. 37 (1) (2010) 13-25.

[16] S.B. Hladky, M.A. Barrand, Fluid and ion transfer across the blood-brain and blood-cerebrospinal fluid barriers; A comparative account of mechanisms and roles, Fluids Barriers CNS 13 (1) (2016) 19.

[17] B.V. Zlokovic, et al., Kinetics of arginine-vasopressin uptake at the blood-brain barrier, Biochim. Biophys. Acta 1025 (2) (1990) 191-198.

[18] B.L. Coomber, P.A. Stewart, Morphometric analysis of CNS microvascular endothelium, Microvasc. Res. 30 (1) (1985) 99-115.

[19] N.R. Saunders, S.A. Liddelow, K.M. Dziegielewska, Barrier mechanisms in the developing brain, Front. Pharmacol. 3 (2012) 46.

[20] C.C. Visser, et al., Characterization and modulation of the transferrin receptor on brain capillary endothelial cells, Pharm. Res. 21 (5) (2004) 761-769.

[21] D. Stern, et al., Receptor for advanced glycation endproducts: a multiligand receptor magnifying cell stress in diverse pathologic settings, Adv. Drug. Deliv. Rev.
54 (12) (2002) 1615-1625

[22] W.M. Pardridge, et al., Evaluation of cationized rat albumin as a potential bloodbrain barrier drug transport vector, J. Pharmacol. Exp. Ther. 255 (2) (1990) 893-899.

[23] W.H. Oldendorf, M.E. Cornford, W.J. Brown, The large apparent work capability of the blood-brain barrier: a study of the mitochondrial content of capillary endothelial cells in brain and other tissues of the rat, Ann. Neurol. 1 (5) (1977) 409-417.

[24] H. Wolburg, A. Lippoldt, Tight junctions of the blood-brain barrier: development, composition and regulation, Vasc. Pharmacol. 38 (6) (2002) 323-337.

[25] G. Bazzoni, E. Dejana, Endothelial cell-to-cell junctions: molecular organization and role in vascular homeostasis, Physiol. Rev. 84 (3) (2004) 869-901.

[26] S. Tsukita, M. Furuse, M. Itoh, Multifunctional strands in tight junctions, Nat. Rev. Mol. Cell Biol 2 (4) (2001) 285-293.

[27] A.W. Vorbrodt, D.H. Dobrogowska, Molecular anatomy of intercellular junctions in brain endothelial and epithelial barriers: electron microscopist's view, Brain Res. Brain Res. Rev. 42 (3) (2003) 221-242.

[28] A.S. Fanning, et al., The tight junction protein ZO-1 establishes a link between the transmembrane protein occludin and the actin cytoskeleton, J. Biol. Chem. 273 (45) (1998) 29745-29753.

[29] A.S. Fanning, et al., The unique-5 and -6 motifs of ZO-1 regulate tight junction strand localization and scaffolding properties, Mol. Biol. Cell 18 (3) (2007) 721-731.

[30] A. Hartsock, W.J. Nelson, Adherens and tight junctions: structure, function and connections to the actin cytoskeleton, Biochim. Biophys. Acta 1778 (3) (2008) 660-669.

[31] M. Aurrand-Lions, et al., Junctional adhesion molecule-C regulates the early influx of leukocytes into tissues during inflammation, J. Immunol. 174 (10) (2005) 6406-6415.

[32] P.F. Bradfield, et al., JAM family and related proteins in leukocyte migration (Vestweber series), Arter. Thromb. Vasc. Biol. 27 (10) (2007) 2104-2112.

[33] R. Daneman, et al., Pericytes are required for blood-brain barrier integrity during embryogenesis, Nature 468 (7323) (2010) 562-566.

[34] M. Hellstrom, et al., Role of PDGF-B and PDGFR-beta in recruitment of vascular smooth muscle cells and pericytes during embryonic blood vessel formation in the mouse, Development 126 (14) (1999) 3047-3055.

[35] P. Lindblom, et al., Endothelial PDGF-B retention is required for proper investment of pericytes in the microvessel wall, Genes Dev. 17 (15) (2003) 1835-1840.

[36] P. Lindahl, et al., Pericyte loss and microaneurysm formation in PDGF-B-deficient mice, Science 277 (5323) (1997) 242-245.

[37] A. Armulik, et al., Pericytes regulate the blood-brain barrier, Nature 468 (7323) (2010) 557-561.

[38] R.D. Bell, et al., Pericytes control key neurovascular functions and neuronal phenotype in the adult brain and during brain aging, Neuron 68 (3) (2010) 409-427.

[39] R. Balabanov, et al., CNS microvascular pericytes express macrophage-like function, cell surface integrin alpha M, and macrophage marker ED-2, Microvasc. Res. 52 (2) (1996) 127-142.

[40] C. Pieper, et al., Brain capillary pericytes contribute to the immune defense in response to cytokines or LPS in vitro, Brain Res. 1550 (2014) 1-8.

[41] A.P. Sagare, et al., Pericyte loss influences Alzheimer-like neurodegeneration in mice, Nat. Commun. 4 (2013) 2932.

[42] J.D. Sengillo, et al., Deficiency in mural vascular cells coincides with blood-brain barrier disruption in Alzheimer's disease, Brain Pathol. 23 (3) (2013) 303-310.

[43] M. Enge, et al., Endothelium-specific platelet-derived growth factor-B ablation mimics diabetic retinopathy, Embo J. 21 (16) (2002) 4307-4316.

[44] F. Pfister, et al., Retinal overexpression of angiopoietin-2 mimics diabetic retinopathy and enhances vascular damages in hyperglycemia, Acta Diabetol. 47 (1) (2010) 59-64.

[45] W.M. Pardridge, Why is the global CNS pharmaceutical market so under-penetrated? Drug Discov. Today 7 (1) (2002) 5-7.

[46] W.M. Pardridge, Alzheimer's disease drug development and the problem of the blood-brain barrier, Alzheimers Dement 5 (5) (2009) 427-432.

[47] W.M. Pardridge, Drug transport across the blood-brain barrier, J. Cereb. Blood Flow Metab. 32 (11) (2012) 1959-1972.

[48] T.T. Wager, et al., Strategies to minimize CNS toxicity: in vitro high-throughput assays and computational modeling, Expert Opin. Drug Metab. Toxicol. 8 (5) (2012) 531-542.

[49] D. Mittal, et al., Insights into direct nose to brain delivery: current status and future perspective, Drug Deliv. 21 (2) (2014) 75-86.

[50] T. Glaser, et al., Targeted nanotechnology in glioblastoma multiforme, Front Pharmacol. 8 (2017) 166.

[51] E. O'Keeffe, M. Campbell, Modulating the paracellular pathway at the blood-brain barrier: current and future approaches for drug delivery to the CNS, Drug Discov. Today: Technol. 20 (2016) 35-39.

[52] Y. Meng, et al., Current and emerging brain applications of MR-guided focused ultrasound, J. Ther. Ultrasound 5 (2017) 26.

[53] J.L. Alves, Blood-brain barrier and traumatic brain injury, J. Neurosci. Res. 92 (2) 2014) 141-147.

[54] B. Fehily, M. Fitzgerald, Repeated mild traumatic brain injury: potential mechanisms of damage, Cell Transpl. 26 (7) (2017) 1131-1155.

[55] R. Prakash, S.T. Carmichael, Blood-brain barrier breakdown and neovascularization processes after stroke and traumatic brain injury, Curr. Opin. Neurol. 28 (6) (2015) 556-564.

[56] M.K. Baskaya, et al., The biphasic opening of the blood-brain barrier in the cortex and hippocampus after traumatic brain injury in rats, Neurosci. Lett. 226 (1) 
(1997) 33-36.

[57] C. Greene, et al., Dose-dependent expressionof claudin-5 is a modifying factor in schizophrenia, Mol. Psychiatry (2017) (in press).

[58] A.J. Becker, Animal models of acquired epilepsy: insights into mechanisms of human epileptogenesis, Neuropathol. Appl. Neurobiol. 44 (Feb. (1)) (2018) 112-129.

[59] L. Librizzi, et al., Cerebrovascular heterogeneity and neuronal excitability, Neurosci. Lett. 667 (February) (2018) 75-83.

[60] M.C. Walker, Pathophysiology of status epilepticus, Neurosci. Lett. 667 (February) (2018) 84-91.

[61] E. Bora, Neurodevelopmental origin of cognitive impairment in schizophrenia, Psychol. Med. 45 (1) (2015) 1-9.

[62] C.Y. Lin, G.E. Tsai, H.Y. Lane, Assessing and treating cognitive impairment in schizophrenia: current and future, Curr. Pharm. Des. 20 (32) (2014) 5127-5138.

[63] S. Najjar, et al., Neurovascular unit dysfunction and blood-brain barrier hyperpermeability contribute to schizophrenia neurobiology: a theoretical integration of clinical and experimental evidence, Front. Psychiatry 8 (2017) 83.

[64] S. Najjar, et al., Neuroinflammation and psychiatric illness, J. Neuroinflamm. 10 (2013) 43.

[65] P.A. Ringen, et al., Increased mortality in schizophrenia due to cardiovascula disease - a non-systematic review of epidemiology, possible causes, and interventions, Front. Psychiatry 5 (2014) 137.

[66] N.A. Uranova, et al., Ultrastructural damage of capillaries in the neocortex in schizophrenia, World J. Biol. Psychiatry 11 (3) (2010) 567-578.

[67] N. Uranova, et al., Reduced capillary density in the prefrontal cortex in schizophrenia, Am. J. Med. Sci. Med. 1 (3) (2013) 45-51.

[68] I. Udristoiu, et al., The microvascular alterations in frontal cortex during treatment with antipsychotics: a post-mortem study, Rom. J. Morphol. Embryol. 57 (2) (2016) 501-506.

[69] V. Vostrikov, D. Orlovskaya, N. Uranova, Deficit of pericapillary oligodendrocytes in the prefrontal cortex in schizophrenia, World J. Biol. Psychiatry 9 (1) (2008) 34-42.

[70] M.J. Webster, et al., Immunohistochemical localization of phosphorylated glial fibrillary acidic protein in the prefrontal cortex and hippocampus from patients with schizophrenia, bipolar disorder, and depression, Brain Behav. Immun. 15 (4) (2001) 388-400.

[71] M.J. Webster, et al., Glial fibrillary acidic protein mRNA levels in the cingulate cortex of individuals with depression, bipolar disorder and schizophrenia, Neuroscience 133 (2) (2005) 453-461.

[72] T.D. Cannon, et al., Cortex mapping reveals regionally specific patterns of genetic and disease-specific gray-matter deficits in twins discordant for schizophrenia, Proc. Natl. Acad. Sci. U. S. A. 99 (5) (2002) 3228-3233.

[73] M.E. Shenton, et al., Abnormalities of the left temporal lobe and thought disorder in schizophrenia. A quantitative magnetic resonance imaging study, N. Engl. J. Med. 327 (9) (1992) 604-612.

[74] J.D. Van Horn, I.C. McManus, Ventricular enlargement in schizophrenia. A metaanalysis of studies of the ventricle:brain ratio (VBR), Br. J. Psychiatry 160 (1992) 687-697.

[75] A. Montagne, et al., Blood-brain barrier breakdown in the aging human hippocampus, Neuron 85 (2) (2015) 296-302.

[76] J. Hua, et al., Abnormal grey matter arteriolar cerebral blood volume in schizophrenia measured with 3D inflow-based vascular-space-occupancy MRI at 7T, Schizophr. Bull. 43 (3) (2017) 620-632.

[77] D. Peruzzo, et al., The impact of schizophrenia on frontal perfusion parameters: a DSC-MRI study, J. Neural Transm. (Vienna) 118 (4) (2011) 563-570.

[78] T.A. Longden, et al., Capillary $\mathrm{K}+$-sensing initiates retrograde hyperpolarization to increase local cerebral blood flow, Nat. Neurosci. 20 (5) (2017) 717-726.

[79] C.S. Wang, et al., Negative symptoms and regional cerebral blood flow in patients with schizophrenia: a single photon emission computed tomography study, Kaohsiung J. Med. Sci. 19 (9) (2003) 464-469.

[80] J. Zhao, et al., The effects of clozapine on cognitive function and regional cerebral blood flow in the negative symptom profile schizophrenia, Int. J. Psychiatry Med. 36 (2) (2006) 171-181.

[81] N.C. Andreasen, C.A. Calarge, D.S. O'Leary, Theory of mind and schizophrenia: a positron emission tomography study of medication-free patients, Schizophr. Bull. 34 (4) (2008) 708-719.

[82] B. Moghaddam, D. Javitt, From revolution to evolution: the glutamate hypothesis of schizophrenia and its implication for treatment, Neuropsychopharmacology 37 (1) (2012) 4-15.

[83] K. Merritt, et al., Nature of glutamate alterations in schizophrenia: a meta-analysis of proton magnetic resonance spectroscopy studies, JAMA Psychiatry 73 (7) (2016) 665-674.

[84] U. Vazana, et al., Glutamate-mediated blood-brain barrier opening: implications for neuroprotection and drug delivery, J. Neurosci. 36 (29) (2016) 7727-7739.

[85] C. Ferrarese, et al., Assessment of reliability and biological significance of glutamate levels in cerebrospinal fluid, Ann. Neurol 33 (3) (1993) 316-319.

[86] R.A. Hawkins, The blood-brain barrier and glutamate, Am. J. Clin. Nutr. 90 (3) (2009) 867S-874S.

[87] Q.R. Smith, Transport of glutamate and other amino acids at the blood-brain barrier, J. Nutr. 130 (4S Suppl) (2000) 1016s-1022s.

[88] R.E. McCullumsmith, et al., Cell-specific abnormalities of glutamate transporters in schizophrenia: sick astrocytes and compensating relay neurons? Mol. Psychiatry 21 (6) (2016) 823-830.

[89] B. Zhang, et al., Common variants in SLC1A2 and schizophrenia: association and cognitive function in patients with schizophrenia and healthy individuals, Schizophr. Res. 169 (1-3) (2015) 128-134.
[90] H. Shalev, Y. Serlin, A. Friedman, Breaching the blood-brain barrier as a gate to psychiatric disorder, Cardiovasc. Psychiatry Neurol. 2009 (2009) 278531.

[91] K. Bechter, et al., Cerebrospinal fluid analysis in affective and schizophrenic spectrum disorders: identification of subgroups with immune responses and bloodCSF barrier dysfunction, J. Psychiatr. Res. 44 (5) (2010) 321-330.

[92] M. Busse, et al., Dysfunction of the blood-cerebrospinal fluid-barrier and N-methyl-D-aspartate glutamate receptor antibodies in dementias, Eur. Arch. Psychiatry Clin. Neurosci. (2017).

[93] D.R. Lara, et al., Increased serum S100B protein in schizophrenia: a study in medication-free patients, J. Psychiatr. Res. 35 (1) (2001) 11-14.

[94] M.L. Schroeter, et al., Serum S100B is increased during early treatment with antipsychotics and in deficit schizophrenia, Schizophr. Res. 62 (3) (2003) 231-236.

[95] M. Wiesmann, et al., Elevated plasma levels of S-100b protein in schizophrenic patients, Biol. Psychiatry 45 (11) (1999) 1508-1511.

[96] M. Rothermundt, et al., Increased S100B blood levels in unmedicated and treated schizophrenic patients are correlated with negative symptomatology, Mol. Psychiatry 6 (4) (2001) 445-449.

[97] M. Rothermundt, et al., S100B serum levels and long-term improvement of negative symptoms in patients with schizophrenia, Neuropsychopharmacology 29 (5) (2004) 1004-1011.

[98] S. Chen, et al., Cognitive dysfunction correlates with elevated serum S100B concentration in drug-free acutely relapsed patients with schizophrenia, Psychiatry Res. 247 (2017) 6-11.

[99] M. Rothermundt, et al., Glial cell dysfunction in schizophrenia indicated by increased S100B in the CSF, Mol. Psychiatry 9 (10) (2004) 897-899.

[100] J. Steiner, et al., S100B serum levels are closely correlated with body mass index: an important caveat in neuropsychiatric research, Psychoneuroendocrinology 35 (2) (2010) 321-324

[101] Y. Iwata, et al., Increased levels of serum soluble L-selectin in unmedicated patients with schizophrenia, Schizophr. Res. 89 (1-3) (2007) 154-160.

[102] J. Masopust, et al., Markers of thrombogenesis are activated in unmedicated patients with acute psychosis: a matched case control study, BMC Psychiatry 11 (2011) 2.

[103] T.T. Nguyen, et al., Abnormal levels of vascular endothelial biomarkers in schizophrenia, Eur. Arch. Psychiatry Clin. Neurosci. (2017).

[104] A. Pillai, et al., Association of serum VEGF levels with prefrontal cortex volume in schizophrenia, Mol. Psychiatry 21 (5) (2016) 686-692.

[105] B.H. Lee, et al., Alterations in plasma vascular endothelial growth factor levels in patients with schizophrenia before and after treatment, Psychiatry Res. 228 (1) (2015) 95-99.

[106] S. Fulzele, A. Pillai, Decreased VEGF mRNA expression in the dorsolateral prefrontal cortex of schizophrenia subjects, Schizophr Res. 115 (2-3) (2009) 372-373.

[107] S. Aboul-Fotouh, N. Elgayar, Atypical antipsychotics such as risperidone, but no paliperidone, worsen vascular endothelial function via upregulation of adhesion molecules VCAM-1, ICAM-1, and E-selectin in diabetic rats, Can. J. Physiol. Pharmacol. 91 (12) (2013) 1119-1126.

[108] E. Domenici, et al., Plasma protein biomarkers for depression and schizophrenia by multi analyte profiling of case-control collections, PLoS One 5 (2) (2010) e9166.

[109] H. Yamamori, et al., Plasma levels of mature brain-derived neurotrophic factor (BDNF) and matrix metalloproteinase-9 (MMP-9) in treatment-resistant schizophrenia treated with clozapine, Neurosci. Lett. 556 (2013) 37-41.

[110] T. Niitsu, et al., A positive correlation between serum levels of mature brain-derived neurotrophic factor and negative symptoms in schizophrenia, Psychiatry Res. 215 (2) (2014) 268-273.

[111] G.M. Khandaker, et al., Inflammation and immunity in schizophrenia: implications for pathophysiology and treatment, Lancet Psychiatry 2 (3) (2015) 258-270.

[112] C. Coisne, B. Engelhardt, Tight junctions in brain barriers during central nervous system inflammation, Antioxid. Redox Signal. 15 (5) (2011) 1285-1303.

[113] B. Engelhardt, L. Sorokin, The blood-brain and the blood-cerebrospinal fluid barriers: function and dysfunction, Semin. Immunopathol. 31 (4) (2009) 497-511.

[114] A. Shimada, S. Hasegawa-Ishii, Histological architecture underlying brain-immune cell-cell interactions and the cerebral response to systemic inflammation, Front. Immunol. 8 (2017) 17

[115] Y. Nakagawa, K. Chiba, Involvement of neuroinflammation during brain development in social cognitive deficits in autism spectrum disorder and schizophrenia, J. Pharmacol. Exp. Ther. 358 (3) (2016) 504-515.

[116] L.W. Harris, et al., The cerebral microvasculature in schizophrenia: a laser capture microdissection study, PLoS One 3 (12) (2008) e3964.

[117] C. D'Mello, M.G. Swain, Liver-brain interactions in inflammatory liver diseases: implications for fatigue and mood disorders, Brain Behav. Immun. 35 (2014) 9-20.

[118] Y. Hwang, et al, Gene expression profiling by mRNA sequencing reveals increased expression of immune/inflammation-related genes in the hippocampus of individuals with schizophrenia, Transl. Psychiatry 3 (2013) e321.

[119] S. Kim, et al., Transcriptome sequencing of the choroid plexus in schizophrenia, Transl. Psychiatry 6 (11) (2016) e964.

[120] P. Katsel, et al., Microvascular anomaly conditions in psychiatric disease. Schizophrenia - angiogenesis connection, Neurosci. Biobehav. Rev. 77 (2017) 327-339.

[121] P. De Rossi, et al., A critical role for VEGF and VEGFR2 in NMDA receptor synaptic function and fear-related behavior, Mol. Psychiatry 21 (12) (2016) 1768-1780

[122] J.F. Enwright Iii, et al., Transcriptome alterations of prefrontal cortical parvalbumin neurons in schizophrenia, Mol. Psychiatry (2017) (in press).

[123] K. Nishiura, et al., PKA activation and endothelial claudin-5 breakdown in the schizophrenic prefrontal cortex, Oncotarget 8 (55) (2017) 93382-93391. 
schizophrenia, Eur. Psychiatry 19 (6) (2004) 354-357.

[125] J. Wei, G.P. Hemmings, A study of the combined effect of the CLDN5 locus and the genes for the phospholipid metabolism pathway in schizophrenia, Prostaglandins Leukot. Essent. Fat. Acids 73 (6) (2005) 441-445.

[126] L. Ye, et al., Further study of a genetic association between the CLDN5 locus and schizophrenia, Schizophr. Res. 75 (1) (2005) 139-141.

[127] N. Wu, et al., A weak association of the CLDN5 locus with schizophrenia in Chinese case-control samples, Psychiatry Res. 178 (1) (2010) 223.

[128] E. Omidinia, et al., Polymorphism of the CLDN5 gene and schizophrenia in an Iranian population, Iran. J. Public Health 43 (1) (2014) 79-83.

[129] H. Ishiguro, et al., Replication study for associations between polymorphisms in the CLDN5 and DGCR2 genes in the 22q11 deletion syndrome region and schizophrenia, Psychiatr. Genet. 18 (5) (2008) 255-256.

[130] K.C. Murphy, L.A. Jones, M.J. Owen, High rates of schizophrenia in adults with velo-cardio-facial syndrome, Arch. Gen. Psychiatry 56 (10) (1999) 940-945.

[131] T. Nitta, et al., Size-selective loosening of the blood-brain barrier in claudin-5deficient mice, J. Cell Biol 161 (3) (2003) 653-660.

[132] V. Postorino, et al., Anxiety disorders and obsessive-compulsive disorder in individuals with autism spectrum disorder, Curr. Psychiatry Rep. 19 (12) (2017) 92.

[133] K. Yenkoyan, et al., Advances in understanding the pathophysiology of autism spectrum disorders, Behav. Brain Res. 331 (2017) 92-101.

[134] A. Masi, et al., An overview of autism spectrum disorder, heterogeneity and treatment options, Neurosci. Bull. 33 (2) (2017) 183-193.

[135] M. Varghese, et al., Autism spectrum disorder: neuropathology and animal models, Acta Neuropathol. 134 (4) (2017) 537-566.

[136] M.V. Lombardo, et al., Hierarchical cortical transcriptome disorganization in autism, Mol. Autism 8 (2017) 29.

[137] E.C. Azmitia, et al., Persistent angiogenesis in the autism brain: an immunocytochemical study of postmortem cortex, brainstem and cerebellum, J. Autism Dev. Disord. 46 (4) (2016) 1307-1318.

[138] W.E. Kaufmann, et al., Autism spectrum disorder in fragile X syndrome: cooc curring conditions and current treatment, Pediatrics 139 (Suppl. 3) (2017) S194-S206.

[139] N. Kabakus, et al., Fragile X syndrome and cerebral perfusion abnormalities: single-photon emission computed tomographic study, J. Child Neurol. 21 (12) (2006) 1040-1046.

[140] A.M. Galvan, R. Galvez, Neocortical vasculature abnormalities in the fragile X mental retardation syndrome, Brain Res. 1471 (2012) 155-161.

[141] A.P. Belagodu, S. Fleming, R. Galvez, Neocortical developmental analysis of vasculature and their growth factors offer new insight into fragile $\mathrm{X}$ syndrome abnormalities, Dev. Neurobiol. 77 (11) (2017) 1321-1333.

[142] C.J. Carter, The barrier, airway particle clearance, placental and detoxification functions of autism susceptibility genes, Neurochem. Int. 99 (2016) 42-51.

[143] Y. Kameno, et al., Serum levels of soluble platelet endothelial cell adhesion molecule-1 and vascular cell adhesion molecule- 1 are decreased in subjects with autism spectrum disorder, Mol. Autism 4 (1) (2013) 19.

[144] C.E. Onore, et al., Levels of soluble platelet endothelial cell adhesion molecule-1 and P-selectin are decreased in children with autism spectrum disorder, Biol. Psychiatry 72 (12) (2012) 1020-1025.

[145] K.J. Tsuchiya, et al., Decreased serum levels of platelet-endothelial adhesion molecule (PECAM-1) in subjects with high-functioning autism: a negative correlation with head circumference at birth, Biol. Psychiatry 62 (9) (2007) 1056-1058.

[146] A.M. Connolly, et al., Serum autoantibodies to brain in Landau-Kleffner variant, autism, and other neurologic disorders, J. Pediatr. 134 (5) (1999) 607-613.

[147] E. Esnafoglu, et al., Evaluation of serum neuron-specific enolase, S100B, myelin basic protein and glial fibrilliary acidic protein as brain specific proteins in children with autism spectrum disorder, Int. J Dev. Neurosci. 61 (2017) 86-91.

[148] L.Y. Al-Ayadhi, G.A. Mostafa, A lack of association between elevated serum levels of S100B protein and autoimmunity in autistic children, J. Neuroinflamm. 9 (2012) 54 .

[149] S.A. Guloksuz, et al., Elevated plasma concentrations of S100 calcium-binding protein B and tumor necrosis factor alpha in children with autism spectrum disorders, Rev. Bras. Psiquiatr. 39 (3) (2017) 195-200.

[150] M.N. Lv, et al., The neonatal levels of TSB, NSE and CK-BB in autism spectrum disorder from Southern China, Transl. Neurosci. 7 (1) (2016) 6-11.

[151] H. Kumar, B. Sharma, Memantine ameliorates autistic behavior, biochemistry \& blood brain barrier impairments in rats, Brain Res. Bull. 124 (2016) 27-39.

[152] H. Kumar, B. Sharma, Minocycline ameliorates prenatal valproic acid induced autistic behaviour, biochemistry and blood brain barrier impairments in rats, Brain Res. 1630 (2016) 83-97.

[153] H. Kumar, B.M. Sharma, B. Sharma, Benefits of agomelatine in behavioral, neurochemical and blood brain barrier alterations in prenatal valproic acid induced autism spectrum disorder, Neurochem. Int. 91 (2015) 34-45.

[154] M. Fiorentino, et al., Blood-brain barrier and intestinal epithelial barrier alterations in autism spectrum disorders, Mol. Autism 7 (2016) 49.

[155] C. Holingue, et al., Gastrointestinal symptoms in autism spectrum disorder: a review of the literature on ascertainment and prevalence, Autism Res. 11 (Jan. (1)) (2018) 24-36.

[156] D.W. Kang, et al., Microbiota transfer therapy alters gut ecosystem and improves gastrointestinal and autism symptoms: an open-label study, Microbiome 5 (1) (2017) 10.

[157] Q. Li, et al., The gut microbiota and autism spectrum disorders, Front. Cell Neurosci. 11 (2017) 120.

[158] R.A. Luna, et al., Distinct microbiome-neuroimmune signatures correlate with functional abdominal pain in children with autism spectrum disorder, Cell Mol Gastroenterol. Hepatol. 3 (2) (2017) 218-230.
[159] R.A. Luna, T.C. Savidge, K.C. Williams, The brain-gut-microbiome axis: what role does It play in autism spectrum disorder? Curr. Dev. Disord. Rep. 3 (1) (2016) 75-81.

[160] D.C. Tarlungeanu, et al., Impaired amino acid transport at the blood brain barrier is a cause of autism spectrum disorder, Cell 167 (6) (2016) 1481-1494 e18.

[161] V.T. Ramaekers, et al., Reduced folate transport to the CNS in female Rett patients, Neurology 61 (4) (2003) 506-515.

[162] G. Novarino, et al., Mutations in BCKD-kinase lead to a potentially treatable form of autism with epilepsy, Science 338 (6105) (2012) 394-397.

[163] R.E. Frye, et al., Cerebral folate receptor autoantibodies in autism spectrum disorder, Mol. Psychiatry 18 (3) (2013) 369-381.

[164] V.T. Ramaekers, et al., Folate receptor autoantibodies and spinal fluid 5-methyltetrahydrofolate deficiency in Rett syndrome, Neuropediatrics 38 (4) (2007) 179-183.

[165] V.T. Ramaekers, et al., Folate receptor autoimmunity and cerebral folate deficiency in low-functioning autism with neurological deficits, Neuropediatrics 38 (6) (2007) 276-281.

[166] R.E. Frye, et al., Blocking and binding folate receptor alpha autoantibodies identify novel autism spectrum disorder subgroups, Front. Neurosci. 10 (2016) 80.

[167] C. Onore, M. Careaga, P. Ashwood, The role of immune dysfunction in the pathophysiology of autism, Brain Behav. Immun. 26 (3) (2012) 383-392.

[168] D.B. Noriega, H.F. Savelkoul, Immune dysregulation in autism spectrum disorder, Eur. J. Pediatr. 173 (1) (2014) 33-43.

[169] T.C. Theoharides, B. Zhang, Neuro-inflammation, blood-brain barrier, seizures and autism, J. Neuroinflamm. 8 (2011) 168

[170] T.C. Theoharides, et al., Mast cell activation and autism, Biochim. Biophys. Acta 1822 (1) (2012) 34-41.

[171] T.C. Theoharides, et al., Mast cells, brain inflammation and autism, Eur. J. Pharmacol. 778 (2016) 96-102.

[172] E. Hendriksen, et al., Mast cells in neuroinflammation and brain disorders, Neurosci. Biobehav. Rev. 79 (2017) 119-133.

[173] T.C. Theoharides, et al., Atopic diseases and inflammation of the brain in the pathogenesis of autism spectrum disorders, Transl. Psychiatry 6 (6) (2016) e844.

[174] B. Vafadari, A. Salamian, L. Kaczmarek, MMP-9 in translation: from molecule to brain physiology, pathology, and therapy, J. Neurochem. 139 (Suppl. 2) (2016) 91-114.

[175] M.W. Abdallah, et al., Amniotic fluid MMP-9 and neurotrophins in autism spec trum disorders: an exploratory study, Autism Res. 5 (6) (2012) 428-433.

[176] H. Sidhu, et al., Genetic removal of matrix metalloproteinase 9 rescues the symptoms of fragile X syndrome in a mouse model, J. Neurosci. 34 (30) (2014) 9867-9879.

[177] R. Bhandari, A. Kuhad, Resveratrol suppresses neuroinflammation in the experimental paradigm of autism spectrum disorders, Neurochem. Int. 103 (2017) 8-23.

[178] M.H. Yoo, et al., Autism phenotypes in ZnT3 null mice: involvement of zinc dyshomeostasis, MMP-9 activation and BDNF upregulation, Sci. Rep. 6 (2016) 28548.

[179] C.G. Gkogkas, et al., Pharmacogenetic inhibition of eIF4E-dependent Mmp9 mRNA translation reverses fragile X syndrome-like phenotypes, Cell Rep. 9 (5) (2014) 1742-1755.

[180] I. Gantois, et al., Metformin ameliorates core deficits in a mouse model of fragile X syndrome, Nat. Med. 23 (6) (2017) 674-677.

[181] R.C. Kessler, et al., Prevalence, severity, and comorbidity of 12-month DSM-IV disorders in the national comorbidity survey replication, Arch. Gen. Psychiatry 62 (6) (2005) 617-627.

[182] G. Lippi, et al., Mental depression and cardiovascular disease: a multifaceted, bidirectional association, Semin. Thromb. Hemost. 35 (3) (2009) 325-336.

[183] H. Liu, et al., Depression after myocardial infarction: TNF-alpha-induced alterations of the blood-brain barrier and its putative therapeutic implications, Neurosci. Biobehav Rev. 37 (4) (2013) 561-572.

[184] D. Ongur, W.C. Drevets, J.L. Price, Glial reduction in the subgenual prefrontal cortex in mood disorders, Proc. Natl. Acad. Sci. U. S. A. 95 (22) (1998) $13290-13295$.

[185] D. Cotter, et al., Reduced neuronal size and glial cell density in area 9 of the dorsolateral prefrontal cortex in subjects with major depressive disorder, Cereb. Cortex 12 (4) (2002) 386-394.

[186] G. Rajkowska, et al., Morphometric evidence for neuronal and glial prefrontal cell pathology in major depression, Biol. Psychiatry 45 (9) (1999) 1085-1098.

[187] R.D. Gosselin, et al., Region specific decrease in glial fibrillary acidic protein immunoreactivity in the brain of a rat model of depression, Neuroscience 159 (2) (2009) 915-925.

[188] M. Banasr, R.S. Duman, Glial loss in the prefrontal cortex is sufficient to induce depressive-like behaviors, Biol. Psychiatry 64 (10) (2008) 863-870.

[189] J. Steiner, et al., Severe depression is associated with increased microglial quinolinic acid in subregions of the anterior cingulate gyrus: evidence for an immunemodulated glutamatergic neurotransmission? J. Neuroinflamm. 8 (2011) 94-94.

[190] P. Gudmundsson, et al., The relationship between cerebrospinal fluid biomarker and depression in elderly women, Am. J. Geriatr. Psychiatry 15 (10) (2007) 832-838.

[191] F. Niklasson, H. Agren, Brain energy metabolism and blood-brain barrier permeability in depressive patients: analyses of creatine, creatinine, urate, and albumin in CSF and blood, Biol. Psychiatry 19 (8) (1984) 1183-1206.

[192] C. Menard, et al., Social stress induces neurovascular pathology promoting depression, Nat. Neurosci. 20 (12) (2017) 1752-1760.

[193] M.L. Schroeter, et al., Serum markers support disease-specific glial pathology in major depression, J. Affect. Disord. 111 (2-3) (2008) 271-280.

[194] M.L. Schroeter, et al., S100B is increased in mood disorders and may be reduced by antidepressive treatment, Neuroreport 13 (13) (2002) 1675-1678. 
[195] M.L. Schroeter, et al., Serum S100B represents a new biomarker for mood disorders, Curr. Drug Targets 14 (11) (2013) 1237-1248.

[196] M.L. Schroeter, J. Steiner, K. Mueller, Glial pathology is modified by age in mood disorders-a systematic meta-analysis of serum S100B in vivo studies, J. Affect. Disord. 134 (1-3) (2011) 32-38.

[197] D.M. Pearlman, et al., Blood levels of S-100 calcium-binding protein B, highsensitivity C-reactive protein, and interleukin-6 for changes in depressive symptom severity after coronary artery bypass grafting: prospective cohort nested within a randomized, controlled trial, PLoS One 9 (10) (2014) e111110.

[198] N. Stroth, P. Svenningsson, S100B interacts with the serotonin 5-HT7 receptor to regulate a depressive-like behavior, Eur. Neuropsychopharmacol. 25 (12) (2015) 2372-2380.

[199] E.S. Wohleb, et al., Stress-induced recruitment of bone marrow-derived monocytes to the brain promotes anxiety-like behavior, J. Neurosci. 33 (34) (2013) 13820-13833.

[200] P. Esposito, et al., Acute stress increases permeability of the blood-brain-barrier through activation of brain mast cells, Brain Res. 888 (1) (2001) 117-127.

[201] Y. Liu, R.C. Ho, A. Mak, Interleukin (IL)-6, tumour necrosis factor alpha (TNFalpha) and soluble interleukin-2 receptors (sIL-2R) are elevated in patients with major depressive disorder: a meta-analysis and meta-regression, J. Affect. Disord. 139 (3) (2012) 230-239.

[202] P. Santha, et al., Restraint stress-induced morphological changes at the blood brain barrier in adult rats, Front. Mol. Neurosci. 8 (2015) 88.

[203] H.S. Sharma, P.K. Dey, Impairment of blood-brain barrier (BBB) in rat by immobilization stress: role of serotonin (5-HT), Indian J. Physiol. Pharmacol. 25 (2) (1981) 111-122.

[204] M. Roszkowski, J. Bohacek, Stress does not increase blood-brain barrier permeability in mice, J. Cereb. Blood Flow Metab. 36 (7) (2016) 1304-1315.

[205] C.L. Raison, L. Capuron, A.H. Miller, Cytokines sing the blues: inflammation and the pathogenesis of depression, Trends Immunol. 27 (1) (2006) 24-31.

[206] P.B. Pun, J. Lu, S. Moochhala, Involvement of ROS in BBB dysfunction, Free Radic. Res. 43 (4) (2009) 348-364.

[207] B. Bauer, A.M. Hartz, D.S. Miller, Tumor necrosis factor alpha and endothelin-1 increase P-glycoprotein expression and transport activity at the blood-brain barrier, Mol. Pharmacol. 71 (3) (2007) 667-675.

[208] A.C. da Fonseca, et al., The impact of microglial activation on blood-brain barrier in brain diseases, Front. Cell Neurosci. 8 (2014) 362.

[209] A.T. Argaw, et al., IL-1beta regulates blood-brain barrier permeability via reactivation of the hypoxia-angiogenesis program, J. Immunol. 177 (8) (2006) 5574-5584.

[210] R. Yirmiya, N. Rimmerman, R. Reshef, Depression as a microglial disease, Trends Neurosci. 38 (10) (2015) 637-658.

[211] G. Rajkowska, et al., Coverage of blood vessels by astrocytic endfeet is reduced in major depressive disorder, Biol. Psychiatry 73 (7) (2013) 613-621.

[212] G. Rajkowska, C.A. Stockmeier, Astrocyte pathology in major depressive disorder: insights from human postmortem brain tissue, Curr. Drug Targets 14 (11) (2013) 1225-1236.

[213] A.J. Thomas, et al., Neuropathological evidence for ischemia in the white matter of the dorsolateral prefrontal cortex in late-life depression, Int. J. Geriatr. Psychiatry 18 (1) (2003) 7-13.

[214] J.J. Miguel-Hidalgo, et al., Vascular and extravascular immunoreactivity for intercellular adhesion molecule 1 in the orbitofrontal cortex of subjects with major depression: age-dependent changes, J. Affect. Disorders 132 (3) (2011) 422-431.

[215] J. Lekakis, et al., Selective serotonin re-uptake inhibitors decrease the cytokineinduced endothelial adhesion molecule expression, the endothelial adhesivenes to monocytes and the circulating levels of vascular adhesion molecules, Int. J. Cardiol. 139 (2) (2010) 150-158.

[216] A. Little, Treatment-resistant depression, Am. Fam. Physician 80 (2) (2009) 167-172.

[217] A.H. Schinkel, P-glycoprotein, a gatekeeper in the blood-brain barrier, Adv. Drug Deliv. Rev. 36 (2-3) (1999) 179-194.

[218] F.E. O'Brien, et al., Interactions between antidepressants and P-glycoprotein at the blood-brain barrier: clinical significance of in vitro and in vivo findings, Br. J. Pharmacol. 165 (2) (2012) 289-312.

[219] T.M. Bruckl, M. Uhr, ABCB1 genotyping in the treatment of depression, Pharmacogenomics 17 (18) (2016) 2039-2069.

[220] T. Fujii, et al., Association between the functional polymorphism (C3435T) of the gene encoding P-glycoprotein (ABCB1) and major depressive disorder in the Japanese population, J. Psychiatr. Res. 46 (4) (2012) 555-559.

[221] B. Breitenstein, et al., ABCB1 gene variants and antidepressant treatment outcome: a meta-analysis, Am. J. Med. Genet. B Neuropsychiatr. Genet. 168b (4) (2015) 274-283.

[222] O.L. de Klerk, et al., Locally increased P-glycoprotein function in major depression: a PET study with $[11 \mathrm{C}]$ verapamil as a probe for P-glycoprotein function in the blood-brain barrier, Int. J. Neuropsychopharmacol. 12 (7) (2009) 895-904.

[223] H. Agren, et al., Low brain uptake of L-[11C]5-hydroxytryptophan in major depression: a positron emission tomography study on patients and healthy volunteers, Acta Psychiatr. Scand. 83 (6) (1991) 449-455.

[224] N.I. Brzozowska, et al., Genetic deletion of P-glycoprotein alters stress responsivity and increases depression-like behavior, social withdrawal and microglial activation in the hippocampus of female mice, Brain Behav. Immun. 65 (2017) 251-261.

[225] O.L. de Klerk, et al., Chronic stress and antidepressant treatment have opposite effects on P-glycoprotein at the blood-brain barrier: an experimental PET study in rats, J. Psychopharmacol. 24 (8) (2010) 1237-1242.

[226] F.E. O'Brien, et al., Inhibition of P-glycoprotein enhances transport of imipramine across the blood-brain barrier: microdialysis studies in conscious freely moving rats, Br. J. Pharmacol. 166 (4) (2012) 1333-1343.

[227] M.R. Ghassemifar, et al., Occludin TM4(-): an isoform of the tight junction protein present in primates lacking the fourth transmembrane domain, J. Cell Sci. 115 (Pt 15) (2002) 3171-3180.

[228] V.S. Subramanian, et al., Tight junction targeting and intracellular trafficking of occludin in polarized epithelial cells, Am. J. Physiol. Cell Physiol 293 (5) (2007) C1717-26.

[229] P. Galecki, et al., Vascular endothelial growth factor gene (VEGFA) polymorphisms may serve as prognostic factors for recurrent depressive disorder development, Prog. Neuropsychopharmacol. Biol. Psychiatry 45 (2013) 117-124.

[230] T. Xie, et al., VEGF-related polymorphisms identified by GWAS and risk for major depression, Transl. Psychiatry 7 (3) (2017) e1055.

[231] A.N. Sharma, et al., Role of trophic factors GDNF, IGF-1 and VEGF in major depressive disorder: a comprehensive review of human studies, J. Affect. Disord. 197 (2016) 9-20.

[232] A.F. Carvalho, et al., Peripheral vascular endothelial growth factor as a nove depression biomarker: a meta-analysis, Psychoneuroendocrinology 62 (2015) $18-26$.

[233] Q.S. Li, et al., Analysis of 23andMe antidepressant efficacy survey data: implication of circadian rhythm and neuroplasticity in bupropion response, Transl. Psychiatry 6 (9) (2016) e889.

[234] K. Gao, et al., Association study of VEGFA polymorphisms with schizophrenia in Han Chinese population, Neurosci. Lett. 590 (2015) 121-125.

[235] K.M. Lin, et al., ABCB1 gene polymorphisms are associated with the severity of major depressive disorder and its response to escitalopram treatment, Pharmacogenet. Genomics 21 (4) (2011) 163-170.

[236] S.H. Lee, et al., MRP1 polymorphisms associated with citalopram response in patients with major depression, J. Clin. Psychopharmacol. 30 (2) (2010) 116-125.

[237] G. Ozbey, et al., ABCB1 C3435T polymorphism is associated with susceptibility to major depression, but not with a clinical response to citalopram in a Turkish population, Pharmacol. Rep. 66 (2) (2014) 235-238.

[238] G. Nikisch, C.B. Eap, P. Baumann, Citalopram enantiomers in plasma and cerebrospinal fluid of ABCB1 genotyped depressive patients and clinical response: a pilot study, Pharmacol. Res. 58 (5-6) (2008) 344-347.

[239] M.C. Rosenhagen, M. Uhr, The clinical impact of ABCB1 polymorphisms on the treatment of psychiatric diseases, Curr. Pharm. Des. 17 (26) (2011) 2843-2851.

[240] B. Breitenstein, et al., The clinical application of ABCB1 genotyping in antidepressant treatment: a pilot study, CNS Spectr. 19 (2) (2014) 165-175.

[241] B. Breitenstein, et al., Association of ABCB1 gene variants, plasma antidepressant concentration, and treatment response: results from a randomized clinical study, J. Psychiatr. Res. 73 (2016) 86-95.

[242] A. Vita, et al., Progressive loss of cortical gray matter in schizophrenia: a metaanalysis and meta-regression of longitudinal MRI studies, Transl. Psychiatry 2 (2012) e190.

[243] Q. Mu, et al., A single $20 \mathrm{mg}$ dose of the full D1 dopamine agonist dihydrexidine (DAR-0100) increases prefrontal perfusion in schizophrenia, Schizophr. Res. 94 (13) (2007) 332-341.

[244] P. Brambilla, et al., Assessment of cerebral blood volume in schizophrenia: magnetic resonance imaging study, J. Psychiatr. Res. 41 (6) (2007) 502-510.

[245] B.M. Cohen, et al., Abnormalities of regional distribution of cerebral vasculature in schizophrenia detected by dynamic susceptibility contrast MRI, Am. J. Psychiatry 152 (12) (1995) 1801-1803.

[246] P. Talati, et al., Increased hippocampal CA1 cerebral blood volume in schizophrenia, Neuroimage Clin. 5 (2014) 359-364.

[247] N. Agarwal, et al., Increased fronto-temporal perfusion in bipolar disorder, J Affect. Disord. 110 (1-2) (2008) 106-114.

[248] J. Kealy, M. Campbell, The blood-brain barrier in glioblastoma: pathology and therapeutic implications, in: A. Tivnan (Ed.), Resistance to Targeted Therapies Against Adult Brain Cancers, Springer Publishing, New York, 2016, pp. 69-87.

[249] A.K. Heye, et al., Assessment of blood-brain barrier disruption using dynamic contrast-enhanced MRI. A systematic review, Neuroimage Clin. 6 (2014) 262-274.

[250] S. Szymanski, et al., Gadolinium-DTPA enhanced gradient echo magnetic resonance scans in first episode of psychosis and chronic schizophrenic patients, Psychiatry Res. 40 (3) (1991) 203-207.

[251] M.K. Winkler, et al., Impaired neurovascular coupling to ictal epileptic activity and spreading depolarization in a patient with subarachnoid hemorrhage: possible link to blood-brain barrier dysfunction, Epilepsia 53 (Suppl. 6) (2012) 22-30.

[252] I. Weissberg, et al., Imaging blood-brain barrier dysfunction in football players, JAMA Neurol. 71 (11) (2014) 1453-1455.

[253] C.A. Gonçalves, M.C. Leite, M.C. Guerra, Adipocytes as an important source of serum S100B and possible roles of this protein in adipose tissue, Cardiovasc. Psychiatry Neurol. 2010 (2010) 790431.

[254] T.A. Pollak, et al., The blood-brain barrier in psychosis, Lancet Psychiatry 5 (Jan. (1) (2018) 79-92

[255] X. Jiao, et al., The role of circulating tight junction proteins in evaluating blood brain barrier disruption following intracranial hemorrhage, Dis. Markers 2015 (2015) 860120

[256] M. Campbell, et al., Targeted suppression of claudin-5 decreases cerebral oedema and improves cognitive outcome following traumatic brain injury, Nat. Commun. 3 (2012) 849.

[257] M. Kinoshita, et al., Targeted delivery of antibodies through the blood-brain barrier by MRI-guided focused ultrasound, Biochem. Biophys. Res. Commun. 340 (4) (2006) 1085-1090.

[258] N. Sheikov, et al., Cellular mechanisms of the blood-brain barrier opening induced by ultrasound in presence of microbubbles, Ultrasound Med. Biol. 30 (7) (2004) 979-989. 
[259] A. Burgess, et al., Focused ultrasound-mediated drug delivery through the bloodbrain barrier, Expert Rev. Neurother. 15 (5) (2015) 477-491.

[260] D.H. Miller, et al., High dose steroids in acute relapses of multiple sclerosis: MRI evidence for a possible mechanism of therapeutic effect, J. Neurol. Neurosurg. Psychiatry 55 (6) (1992) 450-453.

[261] K.G. Blecharz, et al., Glucocorticoid effects on endothelial barrier function in the murine brain endothelial cell line cEND incubated with sera from patients with multiple sclerosis, Mult. Scler. 16 (3) (2010) 293-302.

[262] Z. Zhao, et al., Establishment and dysfunction of the blood-brain barrier, Cell 163 (5) (2015) 1064-1078.

[263] R.D. Bell, et al., Apolipoprotein E controls cerebrovascular integrity via cyclophilin a, Nature 485 (7399) (2012) 512-516. 